\title{
Quantum Gravity and Taoist Cosmology: Exploring the Ancient Origins of Phenomenological String Theory
}

\author{
Steven M. Rosen \\ [This article is slated for Progress in Biophysics and Molecular Biology, \\ Vol. 131 (2017), pp. 34-60. ]
}

\begin{abstract}
In the author's previous contribution to this journal (Rosen 2015), a phenomenological string theory was proposed based on qualitative topology and hypercomplex numbers. The current paper takes this further by delving into the ancient Chinese origin of phenomenological string theory. First, we discover a connection between the Klein bottle, which is crucial to the theory, and the Ho-t'u, a Chinese number archetype central to Taoist cosmology. The two structures are seen to mirror each other in expressing the psychophysical (phenomenological) action pattern at the heart of microphysics. But tackling the question of quantum gravity requires that a whole family of topological dimensions be brought into play. What we find in engaging with these structures is a closely related family of Taoist forebears that, in concert with their successors, provide a blueprint for cosmic evolution. Whereas conventional string theory accounts for the generation of nature's fundamental forces via a notion of symmetry breaking that is essentially static and thus unable to explain cosmogony successfully, phenomenological/Taoist string theory entails the dialectical interplay of symmetry and asymmetry inherent in the principle of synsymmetry. This dynamic concept of cosmic change is elaborated on in the three concluding sections of the paper. Here, a detailed analysis of cosmogony is offered, first in terms of the theory of dimensional development and its Taoist (yin-yang) counterpart, then in terms of the evolution of the elemental force particles through cycles of expansion and contraction in a spiraling universe. The paper closes by considering the role of the analyst per se in the further evolution of the cosmos.
\end{abstract}

KEYWORDS: quantum gravity; Taoism; yin and yang; topology; phenomenological philosophy; physics; cosmogony; string theory; symmetry; dimension

\section{CONTEMPORARY PHYSICS, ARCHETYPAL NUMBERS, AND THE HO-T'U}

In recent decades, there has been little meaningful progress in mainstream physics toward a theory that would effectively unify all the forces of nature-the quantum 
mechanical forces as well as gravitation. Musing ironically on this, physicist Lee Smolin (2006) observed that, "for more than two centuries...our understanding of the laws of nature expanded rapidly.... [yet] today, despite our best efforts, what we know for certain about these laws is no more than what we knew back in the 1970s" (viii). What I demonstrated in my previous contribution to this journal (Rosen 2015 ) is that the crux of the problem lay in quantum mechanics' most basic term: $\hbar$, the quantum of action. Although this indivisible atom of process found at the submicroscopic Planck length of $10^{-35} \mathrm{~m}$ constitutes the cornerstone of more than a century of successful experimentation in physics, it also marks the end of the road for classical analysis, since here the analytical continuity of space gives way to a "graininess" or discreteness that admits of no further quantitative determination. Moreover, the indivisibility of quantized action is closely tied to an indivisibility of observer and observed, and this implicit inseparability of subject and object is anathema to the program of objective analysis that had gone unquestioned for centuries. While physicists have been able to use probabilistic methods to work around the underlying difficulty when their investigations are limited to the quantum mechanical forces, the problem has proven intractable when gravitation must be included in order to realize a comprehensive account of nature.

Reflecting on the persistent failure of physics to arrive at a workable theory of quantum gravity, Smolin called for a different style of doing physics, a "more reflective, risky, and philosophical style" $(2006,294)$ that confronts "the deep philosophical and foundational issues in physics" (290). I applaud this call for a more philosophically oriented physics, and I propose that the stalemate in physics suggests it will no longer be possible for us to rely on the old philosophical foundation. That is because the fusion of subject and object encountered at the heart of quantum physics profoundly conflicts with the currently dominant philosophy of science, presupposing as it does a sharp division of subject and object. In my 2015 essay, I described in detail a new foundation for contemporary physics:

phenomenological philosophy, an approach in which the intimate interaction of subject and object is accepted as fundamental. Although phenomenology remains important in the present paper, it shares the spotlight with a much older philosophical tradition, one that in fact influenced Martin Heidegger and Maurice Merleau-Ponty, two of the leading figures in the phenomenological movement. As philosopher Stephen Priest put it:

Much of what we have thought of as Heidegger's originality is essentially Taoist and Zen and the ramifications of this through Heidegger's influence on Merleau-Ponty's corpus have yet to be identified....Heideggerian 'Modern Continental Philosophy' should no longer be studied in abstraction from its East Asian ground. $(2003,243)^{1}$

The picture of nature advanced by Taoism some twenty-five hundred years ago was of a cosmos in constant cyclical flux. Its ebb and flow of energies was

\footnotetext{
1 In making this assertion, Priest was commenting on Reinhard May's book, Heidegger's Hidden Sources: East Asian Influences on His Work (1996).
} 
expressed by the paradoxical dynamics of two opposing but interpenetrating forces: yang is the active force related to brightness, positivity, expansion and masculinity; yin is the passive medium associated with darkness, negativity, contraction, and femininity. Operating in close harmony, yin and yang generate patterns of change that are specified by the trigrams and hexagrams depicted in the oldest text of classical China: the I Ching or Book of Changes. And the system of transformations set forth in the I Ching is closely related to a primal pattern of action known as the $H o-t^{\prime} u$. In the next section, we will see the significance of the Ho-t'u for contemporary physics, but before I proceed to examine this archetypal configuration, let me clarify my use of the term "archetype," and discuss the general meaning of archetypal numbers.

In the psychology of C. G. Jung, archetypes are structures of the "collective unconscious" imprinted in nature at the deepest level. This was Jung's way of speaking of that which goes beyond the egoic realm of arbitrarily fashioned, finite forms to participate in something more timeless and universal. The prefix archcomes from the Greek archos, a ruler, and appears in such words as "archbishop" and "architect" (master builder), signifying what is chief or principal. Archaeomeans "ancient" and derives from the Greek arché, the beginning, the first. Thus arche denotes what comes first, both in time and importance.

What is the meaning of type? The word stems from the Greek typos, "a blow, the mark of a blow, figure, outline ... from typtein, to beat, strike" (Webster's Unabridged Dictionary). The etymology of "type" therefore suggests action. Putting this together with arche, we can say that an archetype is a primary action pattern, one that is both fundamental and originary. What I am emphasizing here is the dynamic character of the archetype. Therefore, unlike the traditional Platonic sense of archetype as eidos, an absolute, changeless idea or form, I give precedence to viewing the archetype as an elemental process. While it is true that the word "type" has assumed a predominantly static meaning for us-"a symbol; an emblem; a token ... [a] general form, structure, plan ... a model" (Webster's Unabridged Dictionary) my own use of the term archetype retains the decidedly more active sense of the original Greek typos: "from typtein, to beat, strike."

Now, before his death, Jung suggested to his colleague, Marie-Louise von Franz, that the profoundest expression of archetypal truth may be embodied in certain primordial number patterns. This provided the impetus for Number and Time (1974), von Franz's account of number as primal quality, as the underlying, energetic organizing principle of the unus mundus, the cosmic field from which less fundamental archetypes become manifest.

Drawing from a wealth of ancient and medieval Western and non-Western sources, von Franz demonstrates that the contemporary Western notion of number as empty quantity in fact grew out of a very different meaning of number. In building on Jung's characterization of number as the most basic and primitive element of matter and psyche alike, von Franz portrays numbers as "psychophysical patterns of motion" $(1974,74)$. "We are dealing here," says von Franz,

with the principle according to which number, taken qualitatively, is understood to function as a preconscious ... principle of activity; each 
number must be thought of as containing a specific activity that streams forth like a field of force. From this standpoint numbers signify different rhythmic configurations of the [cosmic ground]. (1974, 74-75)

Here the meaning of number differs significantly from the common understanding prevalent today. According to the latter, numbers are generally taken as mathematical values representing particular quantities used for counting and making calculations. While numbers in this sense are certainly indispensable in measuring extrinsic motions, they themselves are completely inert. In contrast, von Franz's archetypal numbers have their own internal rhythms or pulsations, each one constituting a distinctive "phase of transformation" in time $(1974,42)$. Moreover, assembled into certain field configurations or matrices, these numbers constitute nothing less than "cosmic plans" (22). "In the ancient Chinese view," says von Franz, "the universe was 'organized' according to numerical patterns of this kind" (24). Echoing von Franz, let me emphasize that the numbers in question are not mere abstractions but are "psychophysical" in nature. Rather than just being objective, they express the intimate interplay of object and subject also found at the heart of modern physics, with its quantum of action.

Von Franz devotes considerable attention to a particular numerical array, one that is among the most primordial number configurations of archaic China: the "sequence of older heaven" or Ho-t'u (Fig. 1). ${ }^{2}$ This model provides a blueprint for the generation of the trigrams featured in Taoism's I Ching.
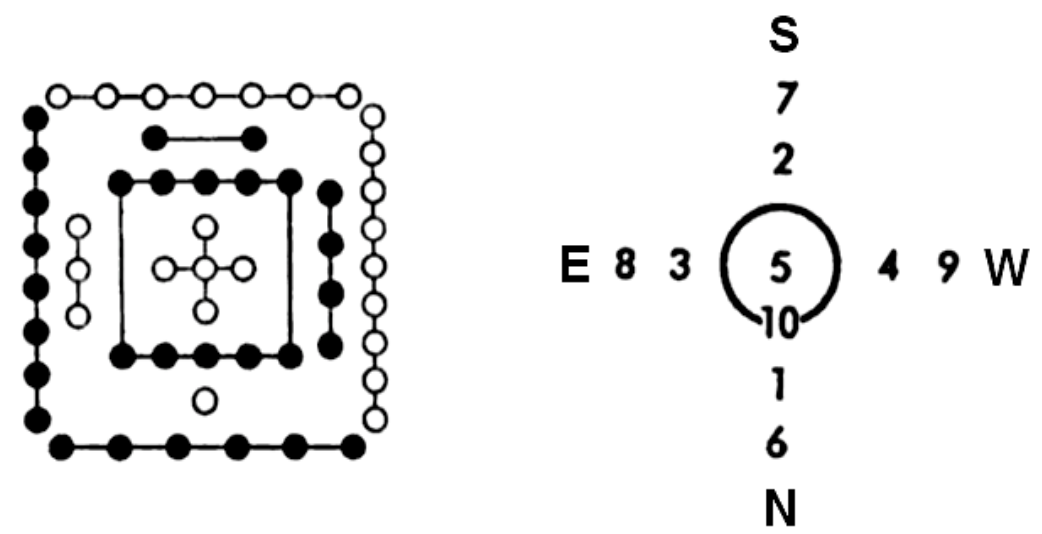

Figure 1. The Ho-t'u number archetype depicted as an array of black and white circles (left) and as a sequence of Arabic numerals (right). (Adapted from von Franz 1974, 23. Source: Granet, Pensée chinoise, (C) Albin Michel 1936, 1968, 130.)

\footnotetext{
${ }^{2}$ In considering the Ho-t'u, Von Franz actually includes an accompanying number array, the Lo-shu or "sequence of younger heaven." This structure is less primordial than the Ho-t'u and thought to derive from it (see 235-241). For our purposes, it will suffice to focus our attention exclusively on the older configuration.
} 
The Ho-t'u sequence consists essentially of a double cycle of numbers, each of which constitutes a rhythmic action unto itself. In the first cycle, there are four such pulsations making up an inner number orbit: 1, 2, 3, 4. The gestural pattern suggested by this is cross-like, and can be taken as following the four cardinal points of the compass - North, South, East, and West, respectively (contra Western convention, the original Chinese diagrams placed North below and South above). Cycle 1 is succeeded by a movement at the center, 5 , after which we shift to the outer number orbit for the second cycle of actions: $6,7,8,9$. These latter pulsations can be seen to correspond to the intercardinal compass points Northwest, Southeast, Northeast, and Southwest, respectively. The sequence ends with a second central pulsation, 10 .

The relationship of the Ho-t'u sequence to the Taoist trigrams is indicated by von Franz as shown in Figure 2.

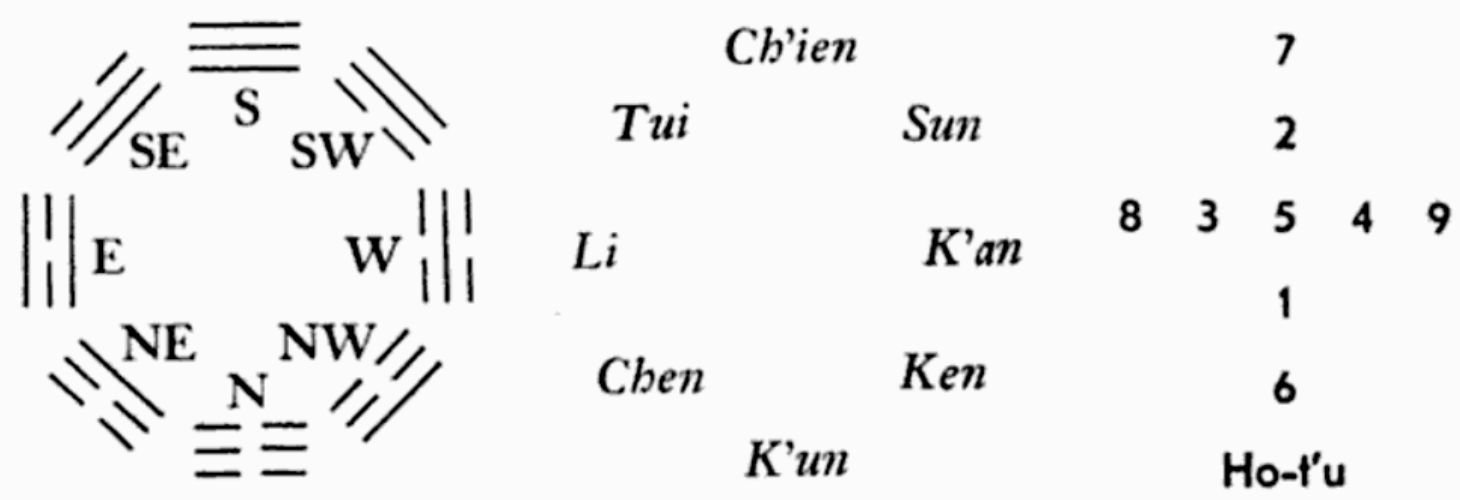

Figure 2. Ho-t'u number archetype (right) and associated trigrams (left), with trigram names displayed in center. (Adapted from von Franz 1974, 25. Source: Granet, Pensée chinoise, (C) Albin Michel 1936, 1968, 148.)

The movement from $K^{\prime} u n(\mathrm{~N})$ to $C h^{\prime} i e n(\mathrm{~S})$ to $\mathrm{Li}(\mathrm{E})$ to $K^{\prime} a n(\mathrm{~W})$ corresponds to the first Ho-t'u cycle (1-4). Von Franz's diagram implies that an action in the unmarked position at the center of the trigram wheel would come next, this being equivalent to position number 5 on the Ho-t'u cross. Then there is the second set of trigram actions-Ken (NW) $\rightarrow$ Tui $(\mathrm{SE}) \rightarrow$ Chen $(\mathrm{NE}) \rightarrow$ Sun $(\mathrm{SW})-$ corresponding to Ho-t'u cycle 2 . The trigram sequence concludes with a final pulsation at the center of the wheel, matching Ho-t'u number 10 (see Fig. 1, right). ${ }^{3}$

Speaking of the Ho-t'u as involving an "internal double movement" (236), Von Franz cites Wilhelm's explanation of a passage from Taoism's I Ching:

"When the trigrams [...] are in motion, a double movement is observable: first, the usual clockwise movement, cumulative and expanding as time goes

\footnotetext{
${ }^{3}$ Commentators have not always been unanimous on the question of how to assign trigrams and their associated compass directions to the numbers of the Ho-t'u. But pursuing debate on this matter is beyond the scope of the present paper.
} 
on, and determining the events that are passing; second, an opposite, backward movement, folding up and contracting as time goes by, through which the seeds of the future take form [...] If we understand how a tree is contracted into a seed, we understand the future unfolding of the seed into a tree." (Wilhelm quoted in von Franz, 1974, 236-237)

A little later in her book, referring to the "Pre-World heavenly order" (another term associated with the Ho-t'u) as comprising a "double internal pattern of motion," von Franz again cites Wilhelm's translation of the I Ching:

"Thunder brings about movement, wind brings about dispersion, rain brings about moisture, the sun brings about warmth." [...] The text continues:

"Keeping Still brings about standstill, the Joyous brings about pleasure, the Creative brings about rulership, the Receptive brings about shelter." As Wilhelm points out, this [latter] passage describes a retrograde movement. In it, the forces of the coming year are unrolled. Pursuance of this line, says Wilhelm, leads to knowledge of the future, the effect of which is prepared in advance by its causes (i.e., seeds), which take shape by contracting. This "retrograde" movement is analogous to the retrograde number steps which, I have postulated, lie behind the qualitative number series. (Von Franz 1974, 243-244)

Von Franz summarizes the Ho-t'u-related "Pre-World" sequence of trigrams as comprising a "rhythmical exchange of powers" in which the "first four inner rhythms are physical (movement, dissolution, moistening, heating) and the second [retrograde] four are psychic (maintenance, rejoicing, mastery, recovery)" (footnote 16, 243-244). For von Franz, this double-cycle "represents an attempt to visualize the whole of psychological and physical existence, the unus mundus, by antithetical time movements and internal rhythms" (245).

What relevance does this ancient cosmology have for the current crisis in modern cosmology, with its problem of quantum gravity? In my 2015 contribution to this journal, I outlined the approach to quantum gravity offered in The SelfEvolving Cosmos (Rosen 2008a). Presently, I am going to build on what I said in 2015 , eventually expanding it to bring out more fully the diachronic aspect, that of cosmic evolution per se. In the course of doing this, we will see how the account of quantum gravity I have proposed is aligned with the "cosmic plans" (von Franz 1974,22 ) implicit in the Ho-t'u and related archetypal structures of Taoist philosophy.

\section{THE TOPOLOGY OF PSYCHOPHYSICAL ACTION}

I mentioned at the outset of the previous section that the sub-microscopic dynamism fundamental to quantum mechanics is $\hbar$, the quantum of action. Let us now consider this concept in greater detail. The formula for $\hbar$ tells us something about its nature: $\hbar=\mathrm{h} / 2 \Pi$. In quantum theory, h is the constant of proportionality 
that relates the energy (E) of a quantum of radiation to the frequency $(v)$ of the oscillation that produced it: $\mathrm{E}=\mathrm{hv}$. Now, if we rewrite this equation by replacing frequency (v) with its inverse, namely, time, we then have $\mathrm{E}=\mathrm{h} / \mathrm{T}$ or $\mathrm{h}=\mathrm{ET}$, and, in physics, energy multiplied by time is a measure of action. Thus h, Planck's constant, gives us quantized action. The angularity of said action, its internal "spin," is expressed by the application of phase, as we see in the formula $\hbar=h / 2 \Pi$. Here $h$ is operated upon by a phase of $2 \Pi$ radians, equivalent to a turn of $360^{\circ}$.

I also mentioned earlier that in quantum mechanics, $\hbar$ is regarded as an indivisible "atom of process," for $\hbar$ is not reducible to smaller units that could be applied in its quantitative analysis. This indivisibility of the quantum domain is closely related to its basic indeterminacy or uncertainty. According to Heisenberg's uncertainty principle, there is a built-in limit to the information we can obtain about the physical properties of quantum systems. This limitation can be stated in terms of Planck's constant: $\Delta \mathrm{p} \Delta \mathrm{q} \approx \hbar$, where $\mathrm{p}$ and $\mathrm{q}$ are variables such as position and momentum, or time and energy (variables that are paired or conjugated so as to be essentially indivisible from each other). The formula says that the product of the uncertainties $(\Delta s)$ of such paired terms approximately equals (cannot be less than) the value of Planck's constant. Clearly then, the phasic indivisibility $(\mathrm{h} / 2 \Pi)$ of Planck-level action is equivalent to its uncertainty $(\Delta \mathrm{p} \Delta \mathrm{q})$.

There is another way to look at the quantum uncertainty. Nearing the submicroscopic Planck length of $10^{-35} \mathrm{~m}$, it appears that precise objective measurement is thwarted by the fact that the energy that must be transferred to a system in order to observe it disturbs that system significantly. This well-known "problem of measurement" in quantum mechanics expresses quantum indivisibility in terms of the indivisibility of the observer and the observed. It seems that in QM, the observer no longer can maintain the classical posture of detached objectivity; unavoidably, $\mathrm{s} /$ he will be an active participant. Evidently this means that quantum mechanical action cannot be regarded merely as objective but must be seen as entailing an intimate merging of object and subject, of matter and psyche, that defies Newtonian order. And the psychophysical character of $\hbar$ brings back to mind the kind of action characteristic of the Ho-t'u number archetype.

Consider further the quantized action of $\hbar$. It takes the form of an odd spinning that Wolfgang Pauli modeled by using complex numbers. Musès (1976) suggested that Pauli's spin matrices for the electron are actually based on a kind of complex number or "hypernumber" that goes beyond Pauli's imaginary $i$ : the hypernumber $\varepsilon$ (defined as $\varepsilon^{2}=+1$, but $\varepsilon \neq \pm 1$ ). What I demonstrate in Cosmos is that the geometric counterpart of $\varepsilon$ is a topological curiosity known as the Klein bottle. This structure plays a highly significant role in Cosmos and in my 2015 paper, and I would now like to ponder it once more in the present context, bringing out its inherently psychophysical nature.

Let us begin with the simple illustration I offered in 2015. 


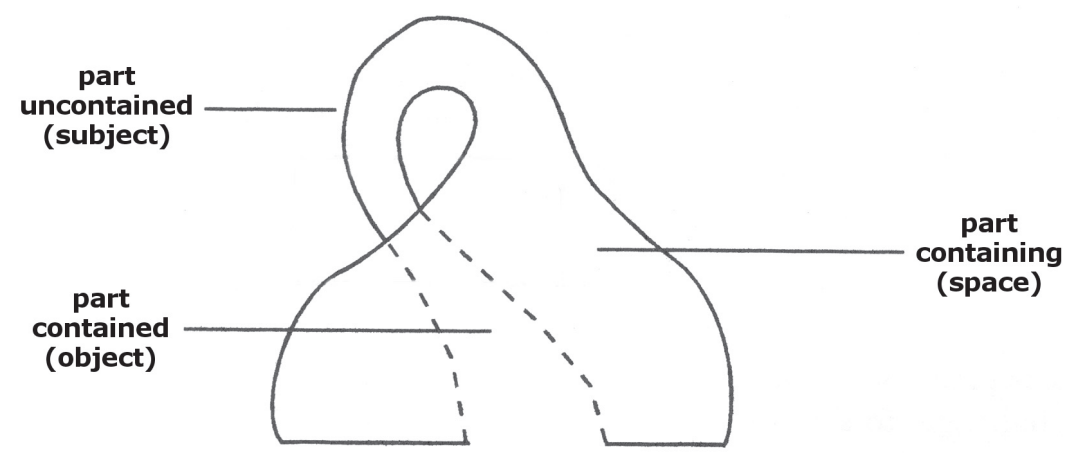

Figure 3. Parts of the Klein bottle (after Ryan 1993, 98)

Figure 3 is my adaptation of communication theorist Paul Ryan's linear schemata for the Klein bottle $(1993,98)$. According to Ryan, the three basic features of the Klein bottle are "part contained," "part uncontained," and "part containing." Here we see how the part contained opens out (at the bottom of the figure) to form the perimeter of the container, and how this, in turn, passes over into the uncontained aspect (in the upper portion of Fig. 3). The three parts of this structure thus flow into one another in a continuous, self-containing movement that flies in the face of the Cartesian division of contained, containing, and uncontainedsymbolically, of object, space, and subject. We can also see an aspect of discontinuity in the diagram. At the juncture where the part uncontained passes into the part contained, the structure must intersect itself. Would this not break the figure open, rendering it simply discontinuous? While this is indeed the case for a Klein bottle conceived as an object in ordinary space, properly seen the Klein bottle actually enacts a dialectic of continuity and discontinuity, as will become clearer in further exploring this peculiar structure. We can say then that, in its highly schematic way, the one-dimensional diagram lays out symbolically the basic terms of a "continuously discontinuous" dialectic involving not only subject and object (psyche and matter), but the mediating space as well. Depicted here is the process by which the three-dimensional object of the sub-microscopic realm, in the act of containing itself, is transformed into the subject. This blueprint for psychophysical interrelatedness gives us a graphic indication of how the mutually exclusive categories of classical thought are surpassed by a relation of mutual inclusion.

The paradoxical nature of the Klein bottle finds expression in its surprising property of one-sidedness. More commonplace topological figures such as the sphere and the torus are two-sided; their opposing sides can be identified in a straightforward, unambiguous fashion. Therefore, they meet the classical expectation of being closed structures, structures whose interior regions ("parts contained") remain interior. In the contrasting case of the Klein bottle, inside and outside are freely reversible. Thus, while the Klein bottle is not simply an open structure, neither is it simply closed, as are the sphere and the torus. In studying the properties of the Klein bottle, we are led to a conclusion that is paradoxical from the classical viewpoint: this structure is both open and closed. The Klein bottle therefore helps to convey to us the fluid relationships between inside and outside, 
closure and openness, continuity and discontinuity that are lost to us in Cartesian experience.

However, must the self-containing one-sidedness of the Klein bottle be seen as involving the spatial container? Granting the Klein bottle's symbolic value, could we not view its inside-out flow from "part contained" to "part containing" merely as a characteristic of an object that itself is simply "inside" of space, with space continuing to play the classical role of that which contains without being contained? In other words, despite its suggestive quality, does the Klein bottle not lend itself to classical idealization as a mere object in space just as much as any other structure?

The schematic representation of the Klein bottle provided by Figure 3 shows that it possesses the curious property of passing through itself. When we consider the actual construction of a Klein bottle in three-dimensional space (by joining one boundary circle of a cylinder to the other from the inside), we are confronted with the fact that no structure can penetrate itself without cutting a hole in its surface, an act that would render the model topologically imperfect (simply discontinuous). So the Klein bottle cannot be assembled effectively when one is limited to three dimensions.

Mathematicians observe that a form that penetrates itself in a given number of dimensions can be produced without cutting a hole if an added dimension is available. The point is imaginatively illustrated by Rudolf Rucker (1977). He asks us to picture a species of "Flatlanders" attempting to assemble a Moebius strip, which is a lower-dimensional analogue of the Klein bottle. Rucker shows that, since the reality of these creatures would be limited to two dimensions, when they would try to make an actual model of the Moebius, they would be forced to cut a hole in it. Of course, no such problem with Moebius construction arises for us human beings, who have full access to three external dimensions. It is the making of the Klein bottle that is problematic for us, requiring as it would a fourth dimension. Try as we might we find no fourth dimension in which to execute this operation.

In contemporary mathematics however, the fact that we cannot create a proper model of the Klein bottle in three-dimensional space is not seen as an obstacle. The modern mathematician does not limit him- or herself to the concrete reality of space but feels free to invoke any number of higher dimensions. Notice though, that in summoning into being these extra dimensions, the mathematician is extrapolating from the known three-dimensionality of the concrete world. This procedure of dimensional proliferation is an act of abstraction that presupposes that the nature of dimensionality itself is not altered. In the case of the Klein bottle, the "fourth dimension" required to complete its formation remains an extensive continuum: an infinitely dense, structureless substrate that itself does not change but that mediates changes in the structured objects it contains (the properties of classical space are examined in depth in Rosen 2008a and 2015). To be sure, the "fourth dimension" is acknowledged as but a formal construct and the Klein bottle is regarded as an abstract mathematical object simply contained in this hyperspace (whereas the sphere, torus, and Moebius strip are relatively concrete mathematical objects, since tangibly perceptible models of them may be successfully fashioned in three dimensions). We see here how the conventional analysis of the Klein bottle unswervingly adheres to the classical formulation of objects in space. Moreover, 
whether a mathematical object must be approached through hyperdimensional abstraction or it is concretizable, the mathematician's attention is always directed outward toward an object, toward that which is cast before his or her subjectivity. This is the aspect of the classical stance that takes subjectivity as the detached position from which all objects are viewed; here, never is subjectivity as such opened to view. Thus the posture of contemporary mathematics is faithfully aligned with that of Descartes and Newton in whatever topic it may be addressing. Always, there is the mathematical object (a geometric form or algebraic function), the analytically continuous space in which the object is contained, and the seldomacknowledged uncontained subjectivity of the mathematician who is carrying out the analysis. And there is never any doubt about the strict separation of these three basic categories.

Now, in his study of topology, Barr advised that we should not be intimidated by the "higher mathematician.... We must not be put off because he is interested only in the higher abstractions: we have an equal right to be interested in the tangible" $(1964,20)$. The tangible fact about the Klein bottle that is glossed over in the higher abstractions of modern mathematics is its hole. Because the standard approach has always presupposed extensive continuity, it cannot come to terms with the inherent discontinuity of the Klein bottle created by its self-intersection. Therefore, all too quickly, "higher" mathematics circumvents this concrete hole by an act of abstraction in which the Klein bottle is treated as a properly closed object embedded in a hyper-dimensional continuum. Also implicit in the mainstream approach is the detached subjectivity of the mathematician before whom the object is cast. I suggest that, by staying with the hole, we may bring into question the classical intuition of object-in-space-before-subject.

Let us look more closely at the hole in the Klein bottle. What kind of hole is it? This loss of continuity is necessary. One certainly could make a hole in the Moebius strip, torus, or any other object in three-dimensional space, but such discontinuities would not be necessary inasmuch as these objects can be properly assembled in space without rupturing them. It is clear that whether such objects are cut open or left intact, the closure of the space containing them will not be brought into question; in rendering these objects discontinuous, we do not affect the assumption that the space in which they are embedded is simply continuous. With the Klein bottle it is different. Its discontinuity does speak to the supposed continuity of three-dimensional space itself, for the necessity of the hole in the bottle indicates that space is unable to contain the bottle the way ordinary objects appear containable. We know that if the Kleinian "object" is properly to be closed, assembled without merely tearing a hole in it, an "added dimension" is needed. Thus, for the Klein bottle to be accommodated, it seems the three-dimensional continuum itself must in some way be opened up, its continuity opened to challenge. Of course, we could attempt to sidestep the challenge by a continuity-maintaining act of abstraction, as in the standard mathematical analysis of the Klein bottle. Assuming we do not employ this stratagem, what conclusion are we led to regarding the "higher" dimension that is required for completing the Klein bottle? If it is not an extensive continuum, what sort of space or dimension is it? 
The question can be addressed by looking again at Figure 3. At first glance, we see a schematic line drawing of a Klein bottle contained within the twodimensional frame of the illustration. But when we consider the actual content of the diagram, we see that, instead of showing the Klein bottle as an object enclosed in space, the diagram portrays space ("part containing") as an aspect of the Klein bottle! What is symbolized schematically in Figure 3 is a dimension of a Kleinian kind, one in which space itself is but one of three ontological terms all of which flow together rather than being split off from one another as happens with the Cartesian paradigm of object-in-space-before-subject. It is clear from this that the Kleinian dimension is not physical but psychophysical, for here subject and object, psyche and physical matter, are mediated by a space in which they permeate each other. So I am proposing that, when the Klein bottle is regarded in concrete terms rather than as a mathematical abstraction, we actually cannot complete its construction as an object in a spatial continuum but are obliged to reformulate dimensionality itself along Kleinian lines. Note that, in this reformulation, the hole in the Klein bottle is neither lightly dismissed in the interest of maintaining continuity, nor does continuity give way to sheer discontinuity. Instead, the very splitting of continuity and discontinuity is called into question and we entertain the proposition that, in completing the Klein bottle, in making it whole, it does not lose its hole. Grasped in this dialectical way, we may say in fact that the Klein bottle constitutes a "(w)hole," a paradoxical structure in which continuity and discontinuity are entwined.

Now, recall our association of the Klein bottle with the microphysical spinning action that lies at the heart of quantum mechanics. This points to the dynamic character of the Kleinian configuration when it is understood in the microworld context. Here, more than a fixed structure, it is an action, a psychophysical atom of process. And when we remember the correlation of Kleinian topology with the hypernumber $\varepsilon$, what comes to mind are the archetypal numbers of the Ho-t'u described by von Franz. ${ }^{4}$ For, as noted earlier, such numbers are "psychophysical patterns of motion" $(1974,74)$ and each number is "thought of as containing a specific activity that streams forth like a field of force" (75). But how specific is the correspondence between the dynamic structure of the Ho-t'u number field and that of the Klein bottle?

In the previous section we found that the Ho-t'u consists of a double cycle of numbers, with each number embodying a rhythmic action unto itself, an internal "phase of transformation," as von Franz termed it $(1974,42)$. The first cycle contains four such phases. This is followed by a return to center (marked by the number 5 in Fig. 1), after which we enter the second cycle of four phases. Here the forward or "clockwise" orientation of the number rhythms in cycle 1 has been reversed, with phases now being characterized as having a backward or retrograde action. And, whereas the "first four inner rhythms are physical...the second four are psychic" (footnote 16, 243-244). Are we to take these two cycles as related in a merely sequential fashion? When the second cycle is enacted is the first cycle simply

\footnotetext{
${ }^{4}$ We know that the Ho-t'u plays a crucial role in the I Ching. Jungian theorist Nathan Schwartz-Salant (2017) relates the I Ching to the Klein bottle by suggesting that the latter is the structure that best expresses the dynamic balance between order and disorder evidenced in the former.
} 
left behind, which would suggest that the cycles are essentially external to each other? It seems that von Franz cannot be suggesting this in view of her emphasis on the thoroughly psychophysical nature of the Ho-t'u number field. At one point she asserts that "number is bound up with the latent material aspect of the psyche and with the latent psychic aspect of matter" (157). Somehow then, latent within the physical circulation of the Ho-t'u (cycle 1) is an aspect of the psychical circulation (cycle 2), and vice versa. Von Franz offers an enigmatic clue for the interconnection of psyche and matter in her discussion of a related mandala, the double wheel of Ezekiel, wherein two wheels are depicted as intersecting each other at right angles (see Fig. 4).

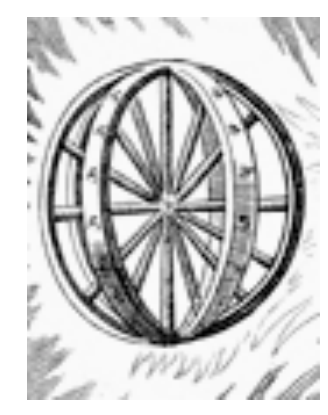

Figure 4. Ezekiel's wheels

The wheels, says von Franz, "do not work in unison but are contiguous at the center, which is a technical impossibility. The two systems are incommensurable" (262). Commenting further, she notes that the "mysterious point of contact between the two systems appears to be the center or a sort of pivot where psyche and matter meet" (263). But the iconic image of Ezekiel's wheels does not in itself effectively indicate the mutual latency of psyche and matter, since the wheels are shown as separate except for the aforementioned point of contact. If the images of the Ho-t'u (Fig. 1) and Ezekiel's wheels (Fig. 4) express the intimate relationship between psyche and matter in merely abstract symbolic terms, is there a deeper, more concretely embodied way of delivering this conjunction? I submit that the hidden topology of the Ho-t'u is none other than that of the Klein bottle. To confirm this, we begin by working with the lower-dimensional counterpart of the Klein bottle: the Moebius strip.

Let us compare the curiously configured Moebius with its more conventional analogue, a cylindrical ring. 


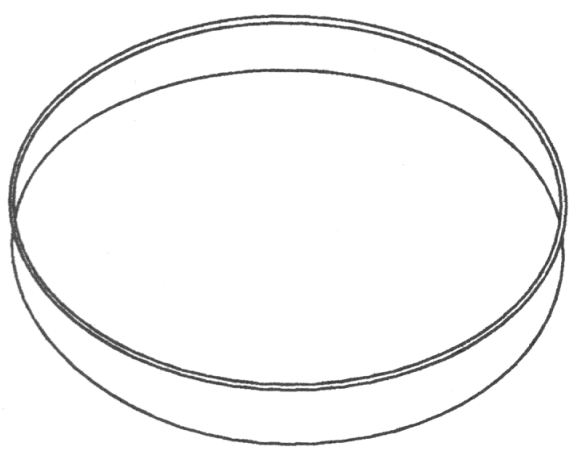

a

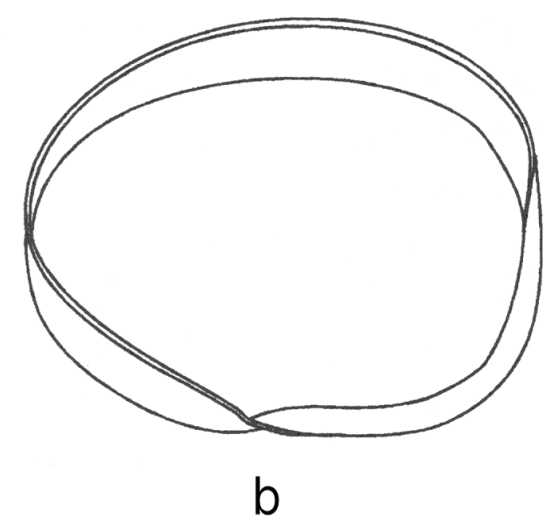

b

Figure 5. Cylindrical ring (a) and Moebius strip (b)

The ordinary ring (Fig. 5a) is constructed by cutting out a narrow strip of paper and joining the ends. To produce the surface of Moebius (Fig. 5b), give one end of the paper strip a half twist (through an angle of $180^{\circ}$ ) before linking it with the other. Now, imagine yourself circling about the cylindrical ring. Positioned on the inner surface of this two-sided structure, you move $360^{\circ}$ around to complete one revolution, returning to your point of departure. Naturally, your passage around the inside of the ring never takes you to the outside. Throughout the journey, you remain on the side on which you began.

It is not like this with movement around the one-sided Moebius surface. While $360^{\circ}$ of revolution do seem to bring you back to your point of origin, at the same time, it is not your point of origin, since you are now on the other side of the surface. Notice the way ordinary circular revolution maintains the simple dichotomy between point of origin and displacement from that point: by $180^{\circ}$ of movement on the cylindrical ring, you are furthest removed from where you began, and by $360^{\circ}$, you are back where you started, the displacement being simply and completely reversed. On the other hand, with $360^{\circ}$ of Moebius revolution, there is a circling back to the point of origin that is at once the point most remote from said origin, since-when sidedness is taken into account-it is the point on the other side of the surface that is diametrically opposed to the starting point. What happens if you continue revolving beyond this $360^{\circ}$ point of quasi-return? After an additional $360^{\circ}$, you find that you have now truly returned, since you have come back to your point of departure on the same side of the surface from which you first set out.

Consider the general resemblance of Moebius action to the action pattern of the Ho-t'u. Both are double cycles involving an initial return to origin (the phase-5 return to center, in the case of the Ho-t'u; see Fig. 1), followed by a second circuit that repeats the process, now completing it in earnest with a second return to origin (Ho-t'u phase 10). However, instead of showing the Ho-t'u as consisting simply of separate cycles connected by a separate thus external inter-cyclical linking phase (phase 5), the one-sidedness of the Moebius superimposes the cycles upon each other, concretely conveying the paradox that these two circulations-the physical and the psychical-also are one. We can say that the cycles of the Moebius are so intimately entwined that the transition to cycle 2 , the new cycle, is just as much a literal repetition of the same cycle. 
A related feature of the Moebius strip further confirms its intimate correspondence with the Ho-t'u. For the latter, we have found that, whereas cycle 1 entails clockwise movement, cycle 2 is counterclockwise. A shift in clock sense is just what takes place in revolving around the Moebius, though the manner of transformation is more subtle than a simple association of one cycle with clockwise action and the other with counterclockwise action. To demonstrate this property, I incorporate a test body into the model: a face in profile (Fig. 6).

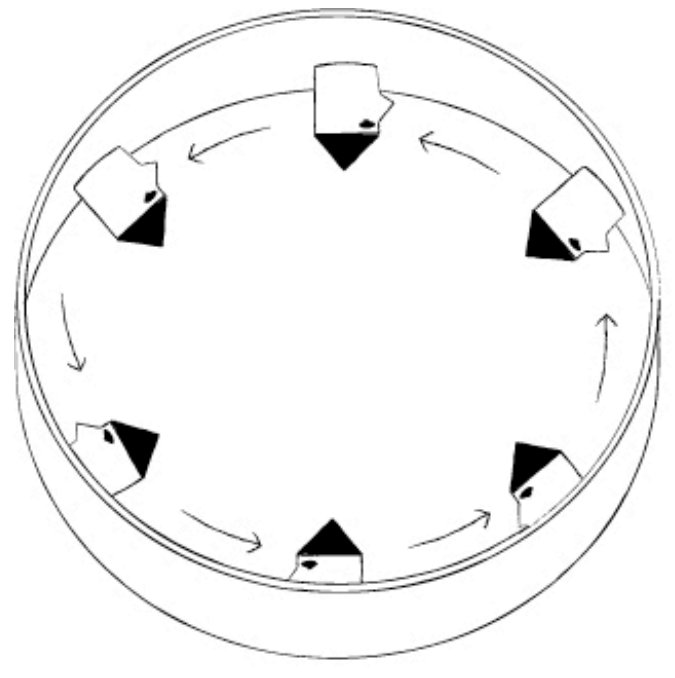

a

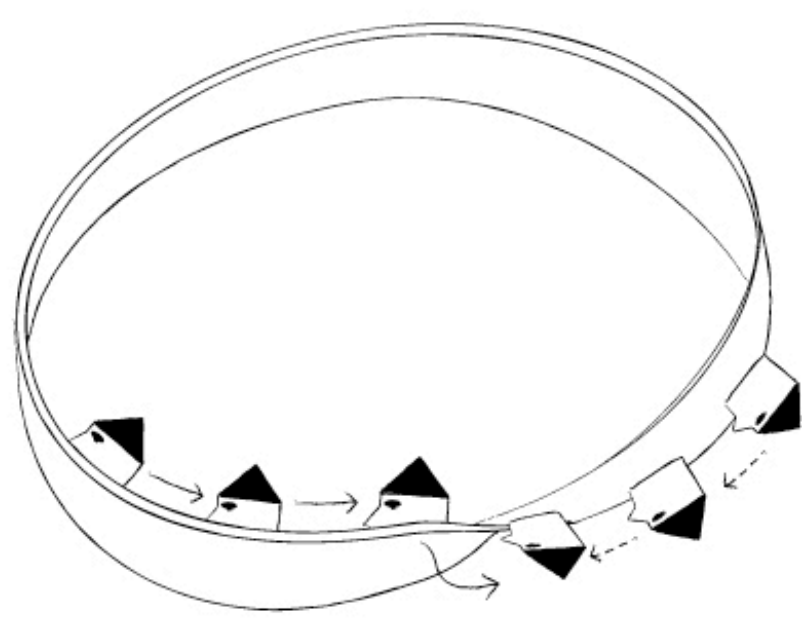

b

Figure 6. Revolution of left-facing profile on cylindrical ring (a) and on Moebius strip (b)

Figure 6a shows a left-facing profile revolving in a counterclockwise direction around a cylindrical ring (see arrows). It is clear that action on the simple ring will continue indefinitely in this manner, with the orientation of the profile never changing (though the half-face is turned upside down). In Figure 6b, we see the profile moving counterclockwise about the Moebius strip. Entering the twist, the left-facing form is changed into its right-facing counterpart, the transformation being completed after $360^{\circ}$ have been traversed. The transformation of left into right therefore coincides with the occurrence of one full cycle of Moebius action. This change in orientation can be seen to reflect a change in clock sense, for, what is counterclockwise to a left-facing profile will be clockwise to one that faces right. Thus, like the action of the Ho-t'u, Moebius action involves a reversal of clock sense.

However, the structure of the Moebius reveals a process of orientation reversal that is more nuanced than what von Franz's text indicates for the Ho-t'u. With the Moebius, it is obvious that cycle 1 action is not uniformly clockwise from beginning to end, this being followed by a shift in gears that gives a second, purely 
counterclockwise cycle. Rather, the clockwise orientation of cycle 1 is fully realized only at the end of the cycle as the culmination of an ongoing transformation from an initial counterclockwise orientation. Then, in entering cycle 2 , the direction of the gear-shift has itself shifted so that the momentum is now from clockwise to counterclockwise. The fact that change in clock sense is inherent to Moebius action means that no separate switching of gears is required to bring it about. The gears are shifting throughout each cycle, with the quasi-return to origin marking the completion of the shift in one direction, and the readiness to begin shifting in the other. The broader implications of these transformations of clock sense will soon be discussed.

Now, while the Moebius strip does generally provide us with an appropriately dynamic and paradoxical model of the Ho-t'u double cycle, the specific phase structure of the Moebius does not meet the requirements of the Hot'u. This observation ties in with the proposition I offered above: it is the Klein bottle that is required for the full embodiment of the Ho-t'u archetypal field. What we will eventually see is that the lower-dimensional Moebius structure properly embodies a lower-order archetypal field.

The limitation of the Moebius with respect to the Ho-t'u can readily be demonstrated. Again, a Moebius strip is produced simply by twisting one end of a narrow band of paper before joining it to the other end. If the continuously curved structure thus created (shown in Figs. 5b and 6b) is flattened out, we obtain the triangular version of the Moebius illustrated in Figure 7.

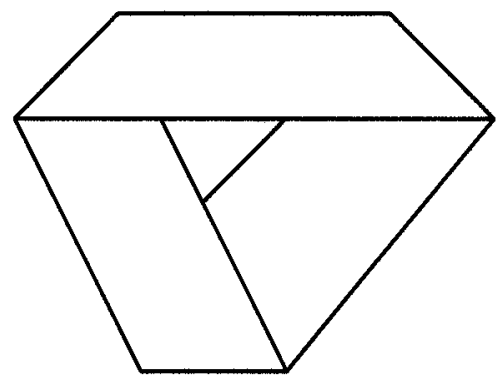

Figure 7. Flattened Moebius strip

The flattening of the Moebius band gives its quantized structure. Instead of each cycle consisting of an unbroken revolution through $360^{\circ}$, now each is composed of distinct phases set off from one another by the creation of edges. For full correspondence to the Ho-t'u, we require two cycles of four phases each. What we have instead is a structure whose two cycles each consist of only three phases.

To bring out the phase structure of the Klein bottle, we begin with a comparison (see Fig. 8 and Fig. 9). 

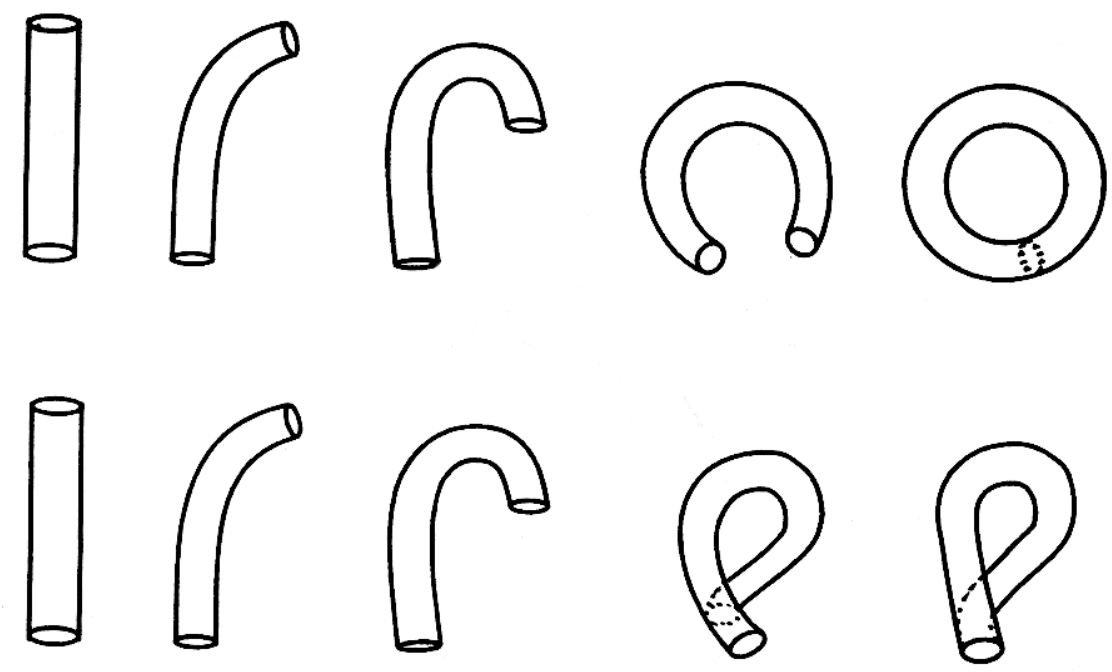

Figure 8. Construction of torus (upper row) and Klein bottle (lower row)

Both rows of Figure 8 depict the progressive closing of a tubular surface that initially is open. In the upper row, the end circles of the tube are joined in the conventional way, brought together through the three-dimensional space outside the body of the tube to produce a torus. By contrast, the end circles in the lower row are superimposed from inside the body of the tube, an operation requiring the tube to pass through itself. This results in the formation of the Klein bottle.

By comparing Figure 8 with Figure 9, we start to see how a quantized version of the Klein bottle can be produced.

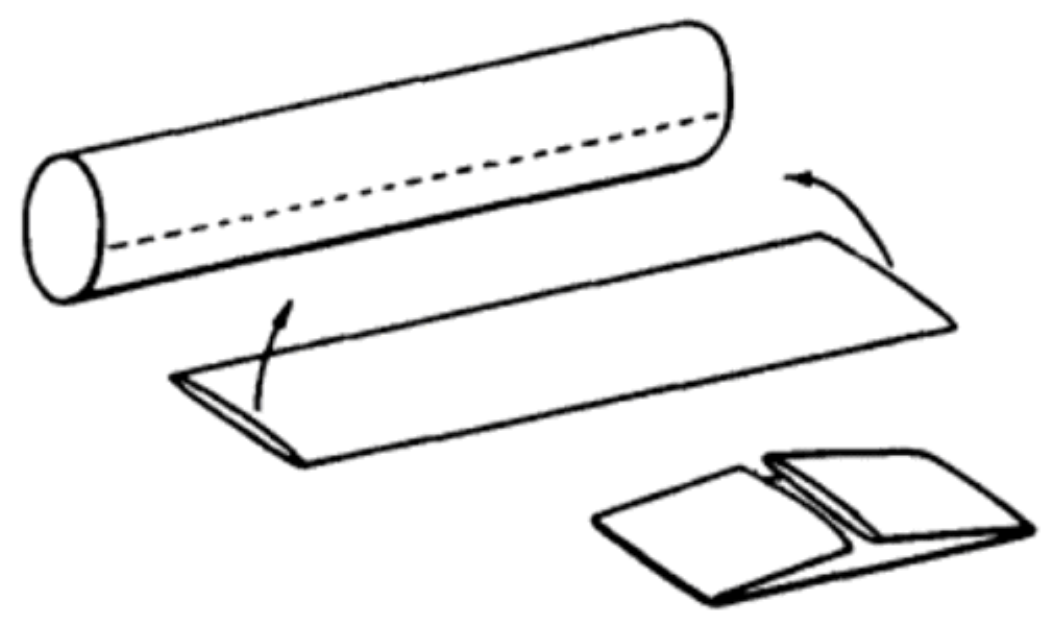

Figure 9. Construction of flat torus (after Barr 1964, 23) (C Courtesy of Dover Publications)

In Figure 9, Barr shows how a long paper cylinder (pictured at the top) can be flattened out along the dashed line, then folded over so that the ends are facing each other. The ends can then be glued together to produce a flat version of the torus. 


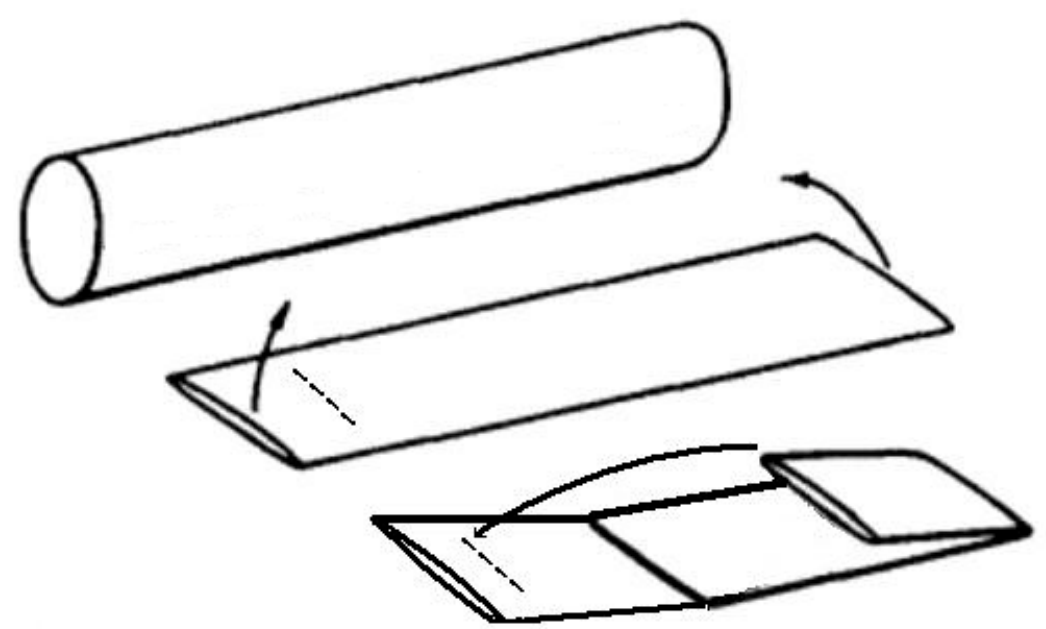

Figure 10. Construction of flat Klein bottle (adapted from Barr 1964, 23)

Figure 10 illustrates the creation of a flat Klein bottle. Once again the paper cylinder is flattened but, this time, we cut a slit near the left end of the cylinder, indicated in the diagram by the dashed line (see middle step of Fig. 10). To complete the construction, we insert the right end into the slit, drawing it through so that it can be brought together with the left end from inside the body of the tube, similar to what happens with the unflattened Klein bottle of Figure 8 when its end circles are superimposed via self-intersection. (The end to be inserted in the slit can be narrowed to allow it to fit.)

By flattening and folding the paper cylinder as we have, eight distinct surfaces have been created. These surfaces, distinguished from one another by the edges created in the folding, give the phases of the quantized Klein bottle, which correspond in their number to the trigram phases of the Ho-t'u. But the one-sided Kleinian configuration is structured more subtly than its two-sided toroidal counterpart, whose four inside surfaces are categorically separated from the four surfaces on the outside. Let us attempt to clarify the Kleinian structure by opening up the folded paper model (Fig. 11). 
CYCLE 1

(PASSAGE FROM INSIDE TO OUTSIDE)
CYCLE 2

(PASSAGE FROM OUTSIDE TO INSIDE)
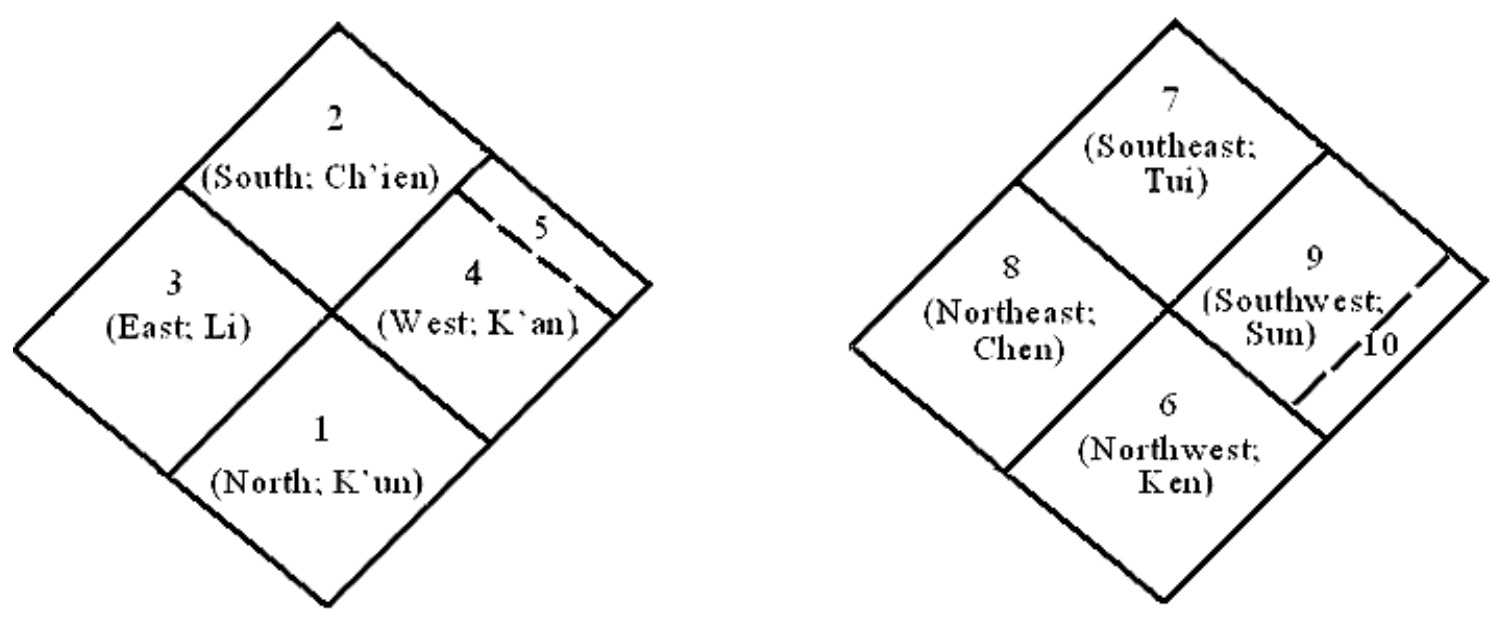

Figure 11. Opened Klein bottle. The folded paper model is opened up to expose its eight subsurfaces. Dashed lines indicate the placement of the slit connecting the two sides of the bottle. Accompanying the Ho-t'u number values are the correlated compass directions and trigram names given in the I Ching (See Fig. 2).

The movement from one surface to another in cycle 1 marks the passage from inside to outside. These quantized transitions are shown as following the pattern of the Ho-t'u cross. With the Ho-t'u (Fig. 2), action is directed from below (North; K'un) to above (South; Ch'ien), thereby establishing the cross' vertical axis. Then, after moving down and to the left (East; $L i$ ), there is a transition to the right (West; K'an) that lays down the horizontal axis, a movement that intersects the vertical. The self-intersection of the Ho-t'u cross that climaxes in passing from the third to fourth stages of action is mirrored on the Klein bottle. Its first three phases are followed by a fourth that brings its self-intersection, as indicated by the dashed line signifying the slit where the surface passes through itself. ${ }^{5}$ And in moving through the slit, we "return to center" (phase 5), whereupon we find ourselves ready to begin again in cycle 2 with phase 6 , though we are now beginning on the outside of the Klein bottle. Note that, unlike the other phases of cycle 1, phase 5 is associated neither with a compass direction nor a trigram. The fifth phase merely marks the completion of cycle 1 by returning us to our point of departure, though now on the opposite side of the surface, and it is this quasi-return that prepares us for initiating the phase actions of cycle 2 .

I want to emphasize that the Klein bottle is indeed one-sided. This means that inside and outside are not strictly separated from each other as with the two-sided

\footnotetext{
${ }^{5}$ Along with the cross, the pelican came to symbolize Christianity in medieval Europe. Like the cross, the pelican was seen as a symbol of self-sacrifice, for the mother of this species was believed capable of feeding her young with her own blood by pressing her bill into her chest. Elsewhere I explored the symbolic relationship between the self-intersecting pelican and the Klein bottle (see Rosen 1995, 2014).
} 
torus but flow into each other in accordance with the bottle's own internal structure. So cycle 1 does not consist of transitions between sub-surfaces all of which simply lie on the outside of the Klein bottle. Instead, movement from surface to surface entails a progressive passage from inside to outside that takes place quite naturally, reaching its culmination with the phase- 5 completion of cycle 1 . It is perhaps easier to see the effect of one-sidedness with the continuous action on the Moebius strip shown in Figures $5 \mathrm{~b}$ and $6 \mathrm{~b}$. Starting from any point on the Moebius, a revolution of $360^{\circ}$ will bring us back to the place where we began (we "return to center") but on the opposite side of the strip.

We have observed as well that Moebius revolution changes clock sense, transforming counterclockwise action into clockwise action and vice versa (leftright orientation is correspondingly transformed; see Fig. 6b). The same applies to action on the Klein bottle. However, even though clock sense changes in cycle 1, we can still associate the overall direction of movement in this cycle with clockwise, given that the momentum is shifting throughout from an initial counterclockwise spinning to a clockwise one. Cycle 1 can thus be said to involve the generation of clockwise action. In relating the quantized Klein bottle to the I Ching, we identify the four specific phases of the cycle- 1 shift to clockwise as the cardinal compass directions of the Ho-t'u: North, South, East, and West.

Cycle 2 reverses the momentum established in cycle 1 . Beginning with phase 6 , our trajectory now takes us from outside to inside and from clockwise to counterclockwise. In view of the way clock sense shifts over the course of this cycle, we can say that the cycle involves the generation of counterclockwise action. Relating this to the I Ching, the four phases of cycle 2 are identified as the intercardinal Ho-t'u directions: Northwest, Southeast, Northeast, and Southwest. (Note, however, that, in section 8, an alternative interpretation of the phases of cycle 2 is offered that departs from I Ching tradition.)

Before going any further, I want to clarify the nature of the rotational action we are dealing with. First of all, Figure 6 shows the relative simplicity of cylindrical action (6a) when compared with action on the Moebius (6b). In the former, the leftfacing profile merely revolves around the length of the ring whereas, in the latter, it also rotates about its own axis. The same combination of rotational components (longitudinal and transverse) is found with Kleinian rotation. It is the transverse component of this topological action that transforms left-right orientation and clock sense. In the case of the action portrayed in Figure 11, jumping from one numbered phase surface to another around the cross is the quantized counterpart of continuous longitudinal revolution on the Klein bottle. The corresponding Ho-t'u diagram shown only in terms of its numerical sequence (Fig. 2, right) shows only this longitudinal component. But when we consider the accompanying trigrams and their compass directions (Fig. 2, left), the transverse component of Kleinian action becomes implicated, with the four directions symbolizing four discrete states in the transition from counterclockwise to clockwise. Thus, coming to understand the hidden topology of the Ho-t'u helps us see more clearly how the Ho-t'u diagram per se and the pulsating trigrams of the I Ching are aspects of the same dynamic structure. 
What are the broader implications of all this? In the present section, I have employed topology to relate the microworld action described in contemporary physics to the action given in ancient China's Ho-t'u number archetype. Although I have presented tangible paper models to detail the manner in which this action takes place, the action itself in fact cannot be realized as an objective event in physical space because it is essentially psychophysical. And as an archetypal action (the prefix archaeo- derives from the Greek arché, the beginning, the first), we may take it as the pre-objective antecedent from which the experience of objective physical reality first arises. This is consistent with the viewpoint expressed by von Franz.

Above I noted von Franz's comments that number archetypes like the Ho-t'u are "cosmic plans" (22), and that "the universe was 'organized' according to numerical patterns of this kind" (24). Considering also that the "first four inner rhythms [of the Ho-t'u] are physical" (footnote 16, 243-244), the Kleinian generation of clockwise action in cycle 1 can be related to the creation of the objective material world. Interesting in this regard is that the first cycle of the Ho-t'u is seen as "cumulative and expanding as time goes on" (236). In cosmogonic terms, this can be associated with the expansion of the physical universe. Here we have "the usual clockwise movement" (236), that in which time flows forward in its natural sequence (Smith et al 1990,118) following time's arrow of increasing entropy associated with cosmic dilation.

After the genesis of clockwise action from an initial counterclockwise orientation in the first Ho-t'u cycle, the momentum shifts back to counterclockwise in the second. Interpreted cosmogonically, this is the backward or retrograde cycle wherein preoccupation with the physical universe projected "out there" in cycle 1 is now overcome and an inward awareness of the psyche is regained-"the second four [inner rhythms] are psychic," says von Franz. But it is not that psyche is favored one-sidedly in this cycle. Instead, the psychophysical nature of the cosmos is realized in full. What had been projected as purely physical or objective is thus presently drawn back in to disclose the archetypal source of said projection, a source that is itself more primordial than the splitting of matter and psyche, object and subject. In drawing inward, the retrograde action of cycle 2 reverses the outthrust of the expanding universe to bring "an opposite, backward movement, folding up and contracting as time goes by" (Wilhelm quoted in von Franz, 236-237). Smith et al, citing a passage from the Shuo-Kua of the I Ching, put it this way: "'going along with ... [time's ordinary] sequence' indicates that one comes to know things in the order that they occur naturally...[whereas] 'going against the direction of time,' means reaching with the mind into the future, against the flow, and knowing things before the stream of time has brought them to us" $(1990,118)$.

I acknowledge the speculative nature of this take on cosmic evolution. In the pages to follow, I will elaborate on my approach, clarifying it, and providing further substantiation. This will involve reprising my demonstration in The Self-Evolving Cosmos (2008a) that an effective rendering of cosmogony and the related issue of quantum gravity requires us to recognize that the Klein bottle is actually but one member of a whole family of interrelated topological structures. In bringing this topological family into play, we will engage with a corresponding family of Taoist 
structures that goes beyond the system of trigrams. In this way, the link between modern Western cosmology and ancient Eastern cosmology will be brought into sharper focus.

\section{QUANTUM GRAVITY AND STRING THEORY}

I opened this paper by noting the lack of progress in theoretical physics toward an account that would unify the four fundamental forces of nature. We found that reconciling the quantum mechanical forces with gravitation requires a theory that can work effectively around the sub-microscopic Planck length of $10^{-35} \mathrm{~m}$.

Indispensable to such a theory is $\hbar$, the quantum of action. This indivisible atom of process constitutes the core of quantum mechanics and can be successfully employed in relatively low energy and low magnification regimes, but dealing with it below the Planck length involves energies so tumultuous that the quantum properties of discontinuity and observer-observed interaction can no longer be managed via probabilistic analysis.

It was in the 1970s, following the progress achieved in unifying the quantum mechanical forces (strong, weak, and electromagnetic), that work on a theory of quantum gravity began in earnest. And this is when confrontation with the chaos of the Planck realm could no longer be avoided. The equations that would unify the four forces were now completely unable to contain the wildly fluctuating Planckian energies, as manifested by the infinite probabilities that turned up to render those equations useless. Movement toward an effective theory of quantum gravity has thus come to a standstill over the past forty years. Commenting on the impasse, Smolin notes that advances have been blocked "despite our best efforts" (2006, viii). What "best efforts" is he referring to?

Since the 1970s, the quest for a mathematical unification of nature has largely been dominated by an approach known as string theory. In this endeavor, the attempt is made to avoid probing below the Planck threshold simply by assuming that the smallest constituents of nature are not indefinitely miniscule point-particles as previous theory had assumed, but string-like vibrating elements of finite extension conveniently scaled at the Planck length. It is because this stratagem has been able to eliminate infinite terms from quantum gravitational equations that it has become the preferred approach. But the price paid for this positivistic ploy has come to be acknowledged (Smolin 2006, Woit 2006). In my own explorations of the matter (Rosen 2004, 2008a, 2008b, 2015), I have identified two problems with string theory.

First, while it is true that string theory serves the classical ontology by sidestepping sub-Planckian ambiguity, an epistemic ambiguity takes its place. String theory's general equations may be free of unmanageable infinities, but theorists must be able to solve these highly abstract equations in a manner that produces a specific description of the world as we know it. As things now stand, the equations yield a vast array of possible solutions with no guiding principle by means of which the field can be narrowed in unique correspondence with known physical reality. A second limitation of the theory is that it seems to contradict itself in its assumption 
of fundamental particles with finite extension. "Strings are truly fundamental," says physicist Brian Greene, "they are 'atoms,' uncuttable constituents" of nature. So, "even though strings have spatial extent, the question of their composition is without any content" $(1999,141)$. But isn't this a contradiction? For-at least according to the classical concept of the continuum not explicitly challenged by string theory, to be spatially extended is to be cuttable, in fact, infinitely divisible. How then could a string be a fundamental particle, an atomic or indivisible ingredient of nature, when it is spatially extended? In sum, string theory is both ambiguous and it appears to contradict itself when seen in classical terms.

But I suggest that despite the limitations of conventional string theory, if we take the vibratory pattern of the fundamental strings as essentially Kleinian in nature-with Kleinian spin not objectified but understood phenomenologically, in psychophysical terms that bridge the gap between subject and object-string theory can gain greater coherence. In fact, I demonstrate in Cosmos that by reformulating the theory in the context of the psychophysical discipline I call topological phenomenology, it can be cast in a form that provides a definitive (albeit qualitative) account of quantum gravity, one that unambiguously yields the fundamental particles of the standard model. We will explore the basis for this conclusion and review its details in coming sections. In the course of doing this, the work of Cosmos will be carried forward and the ancient Chinese roots of phenomenological string theory will be brought to light.

\section{THE TOPODIMENSIONAL FAMILY}

We know that the primary element of action in microphysics is $\hbar$, the quantum of action associated with the emission of radiant energy. We have found as well that the curious spinning accompanying this quantized dynamic is modeled by the hypernumber $\varepsilon$, and that the geometric counterpart of $\varepsilon$ is the Klein bottle. In the form of $\varepsilon \hbar$, the Klein bottle is thus seen to implicitly embody the angular action that lies at the heart of quantum mechanics. Bearing in mind that the Klein bottle cannot be properly understood as a Cartesian object in physical space but must be grasped as inherently psychophysical, I propose we view Kleinian spin as the basic building block of a psychophysical account of quantum gravity.

In Pauli's matrices, $\hbar / 2$ is taken as the fundamental unit of electron spin. In fact, $\hbar / 2$ is the basis for determining the spin of all subatomic particles, fermions and bosons alike. Given the essential role played by spin in quantum mechanics and the underlying significance of the Klein bottle in said spin, I suggested in Cosmos that all microworld dynamics arise from spin of the Kleinian kind: $\varepsilon \hbar / 2$.

Now, in his further exploration of the hypernumber $\varepsilon$, Musès indicated a "higher epsilon-algebra" wherein " $\sqrt{\varepsilon_{n}}$ involves $i_{n}$, the subscripts of course referring to the $(n+1)$ th dimension since $i \equiv i_{1}$ already refers to $D_{2}$ " $(1968,42)$. In view of the intimate relationship between $\varepsilon$ and the Klein bottle, can Musès' implication of a dimensional hierarchy of hypernumber values be given topological expression? The Klein bottle does lend itself to such a generalization. 
Mathematicians have investigated the transformations that result from bisecting topological surfaces. If the Klein bottle is bisected, cut down the middle, it will fall into a pair of oppositely-oriented Moebius strips. Next, bisecting the onesided Moebius strip, a two-sided lemniscatory surface will be produced, its sides being related enantiomorphically (i.e., as mirror opposites). Finally, cutting the lemniscate down the middle yields interlocking lemniscates. The transformation brought about by this bisection is clearly the last one of any significance, since additional bisections - being bisections of lemniscates, can only produce the same result: interlocking lemniscates. The bisection series is completed then when we obtain interlocking lemniscates, a structure termed the sub-lemniscate. By experimenting with the bisection of the Klein bottle in this way, a family of four nested topological structures is discovered (Fig. 12).

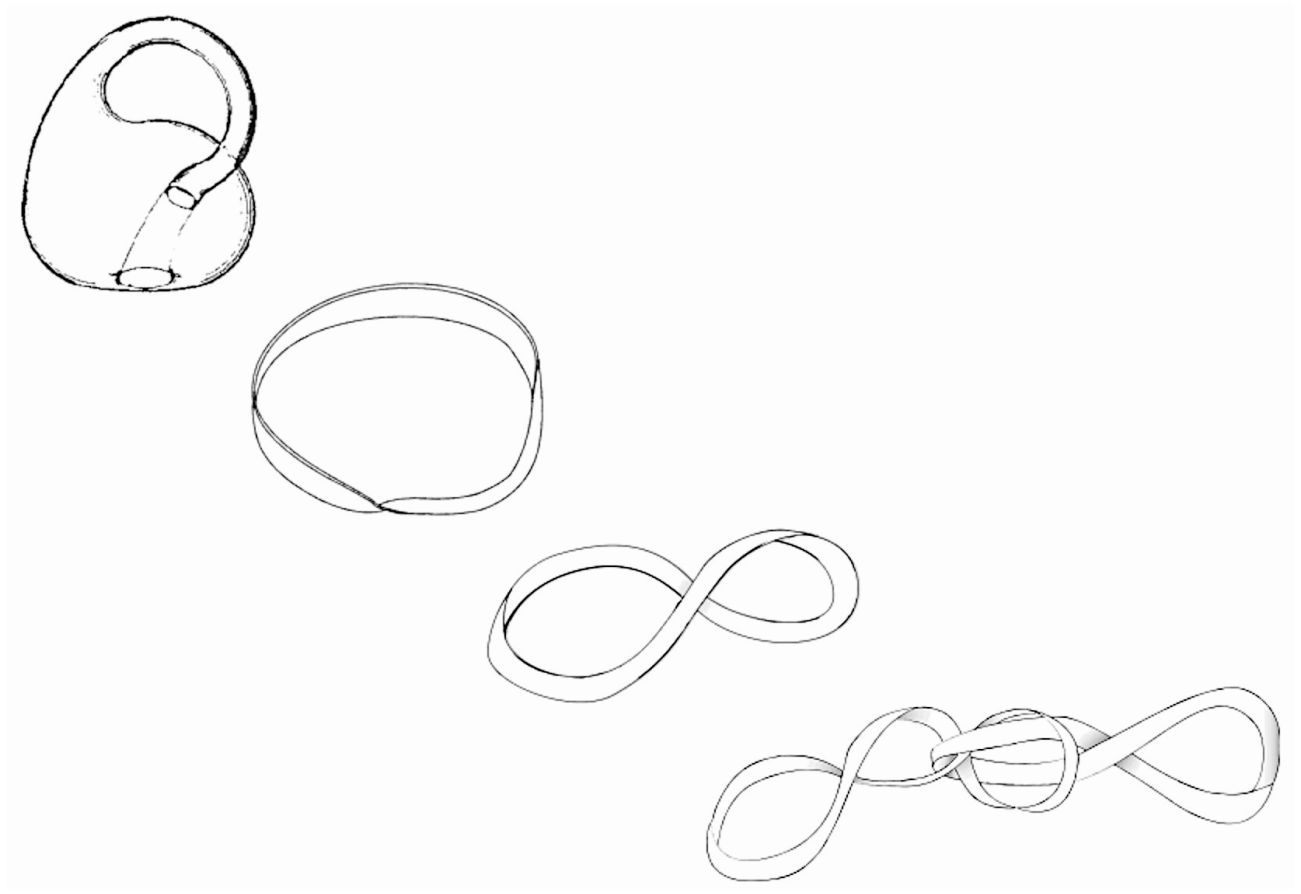

Figure 12. Topological bisection series. From top to bottom: Klein bottle, Moebius strip, lemniscate, sub-lemniscate

In Cosmos, dimensional differences among the four members of the bisection series are studied phenomenologically. While to ordinary observation each member appears as but a two-dimensional surface in three-dimensional space, phenomenological reflection leads to the insight that each actually constitutes a dimensional lifeworld unto itself ("lifeworld" is a phenomenological term for the non-classical, intrinsically psychophysical realm wherein subject and object are intimately entwined). Whereas the Klein bottle is three-dimensional, its nested correlates are of progressively lower dimension: the Moebius is two-dimensional, the lemniscate is one-dimensional, and the sub-lemniscate is zero-dimensional. And, like the Klein bottle, each of these lower-dimensional worlds is a psychophysical realm of action that surpasses the Cartesian division of psyche and matter, subject 
and object. This account of several different topodimensional lifeworlds embedded within each other is consistent with the hierarchy of $\varepsilon$-like spin structures suggested by Musès.

\begin{tabular}{|c|c|c|c|}
\hline$\varepsilon_{\mathrm{D} 0}$ & $\varepsilon_{\mathrm{D} 0} / \varepsilon_{\mathrm{D} 1}$ & $\varepsilon_{\mathrm{D} 0} / \varepsilon_{\mathrm{D} 2}$ & $\varepsilon_{\mathrm{D} 0} / \varepsilon_{\mathrm{D} 3}$ \\
\hline$\varepsilon_{\mathrm{D} 1} / \varepsilon_{\mathrm{D} 0}$ & $\varepsilon_{\mathrm{D} 1}$ & $\varepsilon_{\mathrm{D} 1} / \varepsilon_{\mathrm{D} 2}$ & $\varepsilon_{\mathrm{D} 1} / \varepsilon_{\mathrm{D} 3}$ \\
\hline$\varepsilon_{\mathrm{D} 2} / \varepsilon_{\mathrm{D} 0}$ & $\varepsilon_{\mathrm{D} 2} / \varepsilon_{\mathrm{D} 1}$ & $\varepsilon_{\mathrm{D} 2}$ & $\varepsilon_{\mathrm{D} 2} / \varepsilon_{\mathrm{D} 3}$ \\
\hline$\varepsilon_{\mathrm{D} 3} / \varepsilon_{\mathrm{D} 0}$ & $\varepsilon_{\mathrm{D} 3} / \varepsilon_{\mathrm{D} 1}$ & $\varepsilon_{\mathrm{D} 3} / \varepsilon_{\mathrm{D} 2}$ & $\varepsilon_{\mathrm{D} 3}$ \\
\hline
\end{tabular}

Table 1. Interrelational matrix of topodimensional spin structures

Table 1, the topodimensional spin matrix, gives the $\varepsilon$-based counterpart of the topological bisection series. The three-dimensional Kleinian spinor is written $\varepsilon_{\mathrm{D} 3}$, with lower-dimensional members of the tightly knit spin family designated $\varepsilon_{\mathrm{D} 2}$, $\varepsilon_{D 1}$, and $\varepsilon_{D 0}$ (corresponding to the Moebial, lemniscatory, and sub-lemniscatory circulations, respectively). These terms are arrayed on the principal diagonal of the matrix (extending from upper left to lower right). The interrelationships among the four principal matrix elements, taken two at a time, are reflected in the elements appearing off the main diagonal.

Generally speaking, Table 1 unpacks the dialectical structure of topodimensional interrelations. In keeping with the "musical" implications of string theory, we may regard topodimensional action as inherently vibratory in nature. The principal diagonal of the Table contains a dimensional series of fundamental vibrations or tones, and these four principal terms are coupled to each other two at a time by six pairs of overtone-undertone intervals related to each other in the mirror-opposed fashion of enantiomorphs. The dimensional overtone ratios are the values extending below the fundamental tones, whereas the undertone ratios are the values appearing to the right of the fundamentals. (In Cosmos, the topodimensional action matrix is seen as analogous to the old Pythagorean table, which is portrayed as an expanding series of musical intervals, with fundamental tones on the principal diagonal, flanked by overtones and undertones. For more on this, see section 5.)

Consider in Table 1 the two principal tones of highest dimensionality: $\varepsilon_{\mathrm{D} 2}$ and $\varepsilon_{D 3}$. These matrix elements are linked by the overtone and undertone given in the two corresponding non-principal cells, $\varepsilon_{\mathrm{D} 3} / \varepsilon_{\mathrm{D} 2}$ and $\varepsilon_{\mathrm{D} 2} / \varepsilon_{\mathrm{D} 3}$ (respectively). The enantiomorphically-related coupling cells in question are the hypernumber counterparts of the concretely observable, oppositely oriented Moebius strips which, when glued together, form the Klein bottle. Taken strictly as a principal matrix element, the hypernumber Moebius vibration is the spin structure that constitutes the two-dimensional lifeworld $\left(\varepsilon_{\mathrm{D} 2}\right)$. But when we shift our view of the Moebius, consider it in relation to higher, Kleinian dimensionality, a kind of "doubling" takes place in which the $\varepsilon_{\mathrm{D} 2}$ singular Moebius spin structure becomes a pair of asymmetric, mirror opposed twins, $\varepsilon_{\mathrm{D} 3} / \varepsilon_{\mathrm{D} 2}$ and $\varepsilon_{\mathrm{D} 2} / \varepsilon_{\mathrm{D} 3}$. It is through the fusion of these dimensional enantiomorphs that Kleinian dimensionality is crystallized. Since the Table-1 matrix indicates that all four principal dimensionalities or fundamental tones are interrelated by accompanying off- 
diagonal overtone-undertone pairs, we can draw the general conclusion that higher dimensions emerge through processes of enantiomorphic fusion (this will be spelled out completely in due course).

The process of dimensional generation can be clarified in broad terms by relating it to a reverse movement through the bisection series wherein topological structures are not divided but glued together. To begin, we imagine the fusion of interlocking lemniscates that yields the single lemniscate. This corresponds to the generation of the one-dimensional lifeworld $\left(\varepsilon_{\mathrm{D} 1}\right)$. Next, we picture the enantiomorphically-related sides of the two-sided lemniscate merging to form the one-sided Moebius structure, this being associated with the genesis of the twodimensional lifeworld $\left(\varepsilon_{\mathrm{D} 2}\right)$. Finally, we imagine Moebius enantiomorphs fusing to produce the Klein bottle, which corresponds to the evolution of our threedimensional lifeworld $\left(\varepsilon_{\mathrm{D} 3}\right)$. With each fusion, a lower-dimensional lifeworld is absorbed by a world of higher dimension, taken into it in such a way that the lower dimension is concealed. In the end, we have three lower-dimensional vibratory structures concealed within the three-dimensional Kleinian vibration, much as lower dimensions are hidden by becoming "curled up" within visible $3+1$ dimensional space-time in the conventional string theoretic account of dimensional cosmogony. It turns out, in fact, that the phenomenological approach arrives at the same total number of dimensions as does the conventional theory.

What I demonstrate in Cosmos is that the Kleinian spinor, $\varepsilon_{\mathrm{D} 3}$, is not itself an extended three-dimensional space, but is a quantized three-dimensional blend of space and time that first gives birth to our familiar $3+1$-dimensional space-time. ${ }^{6}$ In like manner, the two-dimensional Moebius spinor $\left(\varepsilon_{\mathrm{D} 2}\right)$ spins out a $2+1$ dimensional space-time, the lemniscatory spinor $\left(\varepsilon_{\mathrm{D} 1}\right)$ sends forth a $1+1$ dimensional space-time, and the sub-lemniscatory spinor $\left(\varepsilon_{\mathrm{D} 0}\right)$ projects a $0+1$ dimensional space-time. A simple summation of projected space-time dimensions gives us a total of ten, with the six lower dimensions- $(2+1)+(1+1)+(0+1)-$ being hidden within the larger $3+1$-dimensional space-time. This picture of overall ten-dimensionality, with six dimensions concealed, accords with the basic account provided by orthodox string theory. Thus we may say that our four phenomenological spinors spin out the ten space-time dimensions of string theory. ${ }^{7}$

Yet despite the general agreement on the number of space-time dimensions, important differences exist between conventional and phenomenological interpretations of string theory. Mainstream theorists have approached cosmogony by adopting the concept of symmetry breaking. In this narrative, the four forces of nature are conceived as vibrating strings that initially existed in a purely symmetric ten-dimensional space scaled around the Planck length. Subsequently, the perfect primordial symmetry was spontaneously broken by a dimensional bifurcation in which four of the original dimensions expanded to produce the visible universe we

\footnotetext{
6 The Kleinian spinor is a "natal space," a "matrix [for...] existing space," to use Merleau-Ponty's way of describing the generative role played by phenomenological dimensionality $(1964,176)$.

7 With the extension of string theory known as $M$-theory, eleven dimensions are actually entailed, though the eleventh dimension is not like the other ten. This "extra" dimension in fact may be interpreted in phenomenological terms. See The Self-Evolving Cosmos.
} 
know today, with the other dimensions remaining hidden. Coupled with this was the breaking of force-field symmetry to create the appearance of irreconcilable differences among the forces.

However, while the foregoing account of cosmogony incorporates both dimensional and force-field symmetry breaking, the two are not precisely aligned with each other in the theoretical reckoning. This reflects the fact that contemporary theorists have been unable to articulate a complete geometric rendering of cosmic evolution. For the geometric program fully to be realized, the physical events described in the standard and inflationary models of cosmic development would need to be specifically expressible as dimensional events. What Heinz Pagels noted twenty years ago in discussing the extra-dimensional (Kaluza-Klein) interpretation of cosmogony remains true today: "No one has yet been able to find a realistic Kaluza-Klein theory which yields the standard model" $(1985,328)$. In the stringtheoretic application of Kaluza-Klein theory, one obvious reason for this limitation is the absence of a conceptual principle that could guide the analyst to unambiguous solutions of the ten-dimensional general equations, solutions specifying the exact shapes of the hidden dimensions that would correspond to the physical facts of the standard model. Of course, if the prevailing theory cannot tell us what the dimensional structures are that correspond to physical reality, it can hardly inform us on how these dimensions develop. In point of fact, there is really no positive feature intrinsic to the theory that provides for the evolution of dimensions. From what I can tell, the only reason dimensional bifurcation is assumed to have taken place at all is that theorists must somehow account for the present inability to observe six of the ten dimensions needed for a consistent rendering of quantum gravity (one that avoids untenable probability values).

Smolin seems to put his finger on the underlying problem in calling attention to the "wrong assumption" physicists "are all making" when they present the "whole history of constant motion and change...as something static and unchanging" (2006, 256-57). When authentic change is thus denied, it is not surprising that no natural, parsimonious way of accounting for cosmogony is forthcoming. Conventional string theory well exemplifies this adherence to the classical intuition of changelessness in the primacy it gives to the notion of symmetry. It is in assuming an initial state of "perfect symmetry" that theorists must resort to the artifice of "spontaneous symmetry breaking," an alleged event that-far from being a natural consequence of the purely symmetric theory-is gratuitously invoked without a compelling explanation of its basis.

The inherent dynamism of phenomenological string theory affords a way out of the impasse. Instead of artificially appending asymmetry to a primordially perfect symmetry, a dialectic of symmetry and asymmetry is offered that permits an unequivocal, intrinsically meaningful account of the evolving forces of nature. This principle of "synsymmetry" (Rosen 1975, 1994, 2006, 2008a) is implicit in the topological bisection series and its associated topodimensional spin matrix (Table 1).

For a simple illustration, consider the Moebius strip. It arises from the fusion of mirror-opposed, asymmetrically-related sides of the lemniscate. We can say that, through this union of opposites, the asymmetry of lemniscatory sides is rendered 
symmetric. However, while the Moebius can be deemed symmetric vis-à-vis the fused lemniscatory sides that constitute it, at the same time it is itself a member of an enantiomorphically asymmetric pair whose own fusion produces the Klein bottle. We may generally conclude that the members of our topodimensional family are neither simply asymmetric nor simply symmetric, but synsymmetric: a given member combines symmetry and asymmetry in such a way that it is symmetric in relation to its lower-dimensional counterpart and asymmetric in relation to its higher one (the sub-lemniscate is an exception to this, since it has no lowerdimensional counterpart). I propose that the synsymmetry concept, viewed dynamically in terms of enantiomorphic fusion events, constitutes a guiding principle for cosmogony. The forces and particles of nature evolve by a general process wherein asymmetric dimensional enantiomorphs fuse to create a dimensional symmetry that at once inherently gives way to new asymmetry. My topo-phenomenological interpretation of cosmogony is elaborated in sections 6-9, where a lot more detail is given on dimensional development, cosmogony by enantiomorphic fusion, and quantum gravity. Indeed, a lot more detail will be needed.

Recall that, in the course of examining the relationship between the Klein bottle and the Ho-t'u number archetype in section 2, I offered a preliminary glimpse of the cosmogonic implications of my account. Since the topodimensional explanation of cosmogony just given does not address those implications completely, it will be necessary for me to expand on what I have said here. Only when further detail has been disclosed on the specific nature of cosmic evolution will we be able to fully appreciate the connection between what was intimated in section 2 and what has been set forth in the present section. And this, in turn, will allow us to grasp the full relationship between topodimensional and Taoist approaches to cosmogony. However, before carrying out the requisite elaboration, I want to demonstrate that the topological family forming the core of phenomenological string theory has its counterpart in ancient China.

\section{THE WORLD'S OLDEST STRING THEORY}

Music has long ... provided the metaphors of choice for those puzzling over questions of cosmic concern. From the ancient Pythagorean "music of the spheres" to the "harmonies of nature" that have guided inquiry through the ages, we have collectively sought the song of nature in the gentle wanderings of celestial bodies and the riotous fulminations of subatomic particles. With the discovery of superstring theory, musical metaphors take on a startling reality, for the theory suggests that the microscopic landscape is suffused with tiny strings whose vibrational patterns orchestrate the evolution of the cosmos. (Greene 1999, 135)

I demonstrated in section 2 that the hidden topology of ancient China's Ho-t'u number archetype is Kleinian in nature. In the previous section, we found that the Klein bottle does not stand alone but is a member of a closely-knit family of four topological structures constituting the core of phenomenological string theory. 
What we are presently going to see is that the Ho-t'u and its associated trigram system also participate in a larger fourfold grouping, one that comprises the "string theory" from which phenomenological string theory can be said to have originated.

I noted earlier that the vibrations or tones of the topodimensional action matrix (Table 1) bear a resemblance to the tonal system set forth in the old Pythagorean table. This table is also known as the Lambdoma (since it is often shown in a configuration suggestive of the Greek letter lambda), and it is connected to the idea of the "music of the spheres." The first four intervals of the Pythagorean table are displayed in Table 2.

\begin{tabular}{|l|l|l|l|}
\hline $0 / 0$ & $0 / 1$ & $0 / 2$ & $0 / 3$ \\
\hline $1 / 0$ & $1 / 1$ & $1 / 2$ & $1 / 3$ \\
\hline $2 / 0$ & $2 / 1$ & $2 / 2$ & $2 / 3$ \\
\hline $3 / 0$ & $3 / 1$ & $3 / 2$ & $3 / 3$ \\
\hline
\end{tabular}

Table 2. Section of the Pythagorean table

The table is usually portrayed as an indefinitely expanding series of musical intervals, but there are many variations of it in the literature, with some excluding the $0 / 0$ interval entirely or placing it outside the body of the table (see, for example, Godwin 1989, 95). What is shown in the representation of the table I have employed is a set of relationships that mirrors our topodimensional action matrix (Table 1). There is a principal diagonal the first tone of which is $0 / 0$, seen in the upper lefthand corner; this corresponds to $\varepsilon_{\mathrm{D} 0}$, the zero-dimensional term of Table 1 . I will have more to say about $0 / 0$ below. The $0 / 0$ fundamental tone is followed on the main diagonal by a series of non-zero fundamentals constituting reflexive, selfdivisive "forms of unity" (Musès, 1968, 32). That is, each non-zero fundamental can be taken as a self-division that by ordinary arithmetic would equal 1 but that is nevertheless distinctive in the Pythagorean context: $1 / 1 \neq 2 / 2 \neq 3 / 3$. This series of self-divisions may be related to the topological series of self-intersecting forms pictured in Figure 12, and it is implicit as well in the values of Table 1. Note also that, in both Tables 1 and 2, the four principal terms are coupled to each other two at a time by six enantiomorphically related pairs of overtone-undertone values. We may therefore entertain the proposition that the values provided in Table 1 are the topodimensional counterparts of the Pythagorean musical intervals appearing in Table 2. Accordingly, these rhythmical relationships can be said to give us the "music of the dimensional spheres." 
Musicologist Joscelyn Godwin asserts that the Pythagorean table is "an image of the universe" $(1992,191)$ and "a means toward symbolic explanation and possible illumination concerning cosmic and metaphysical realities" (190). Musicologist Rudolf Haase similarly emphasizes the spiritual and cosmogonic implications of the Lambdoma, intimating that it may be nothing less than an archetypal ground plan for the creation of all that is:

It is well known that the construction of the world on the basis of two antithetical principles is an age-old concept: it is best known as the yang and yin of Chinese tradition, but also forms an important element of Pythagorean philosophy, which is why Kayser [Haase's teacher] revived this dualism in referring to the similarly dual structure of the Lambdoma, regarding it as the foundation of the latter. (Haase, 1989, 103)

For her part, von Franz observes that the "ancient Chinese...divided their whole psychophysical cosmos into a periodic twofold rhythm, a reciprocal...YinYang motion. Yin and Yang are...a symphony of alternating rhythms in which spatial elements (in front-behind) and temporal elements (before-after) are not separable." Von Franz goes on to cite Marcel Granet's musical simile wherein "Yin and Yang play in concert (tiao) and harmonize (ho). "The whole universe has a rhythmical basic structure'"' $(1974,95)$. In a footnote on the same page, von Franz suggests that "Ho means the harmony of a piece of music." And in that same context, she speaks of the "rhythmical internal movement" found in the "older heavenly order," the order related to the Ho-t'u. Later, after referring to field-like Chinese number matrices "which served as rhythmically organized...images of the aspects of the cosmic whole," she notes the possibility that "the famous Pythagorean lambdoma ...also was originally a field arrangement in circular form" $(1974,147)$.

The foregoing reflections on the Pythagorean table and the harmonies of yin and yang offer an intimation of the primordial "string theory" on which our topophenomenological/psychophysical string theory is based. The Ho-t'u number archetype is of course part of this ancient symphony. But we have seen that the trigram system associated with the Ho-t'u maps onto the Klein bottle and the latter is just one member of a whole topological family. We are now prepared to look at the corresponding yin-yang family of which the Ho-t'u trigram system is but one member.

In section 1, I introduced the cosmology of Taoism by describing its vision of a universe in constant flux. Philosopher Jeaneane Fowler develops this theme at length, beginning with the observation that "that which informed all the transformations in the cosmos was posited as Tao" $(2005,46)$. Citing the I Ching, Fowler further asserts that the Tao is

"the immutable, eternal law at work in all change...the principle of the one in the many." ... [Tao] is that which generates the tension between opposites, that which makes changes and transformations possible, and the power that renews that tension from moment to moment. It is the quiet, spontaneous 
power that eternally gives energy to the cosmos, to the rhythmic composition of the stars and planets as much as to the energy that a tiny seed needs for germination. (46)

Later in her text, Fowler corrects the earlier impression that the Tao is associated exclusively with the One, for "Tao is beyond even the One" (76). Indeed, we really cannot say what the Tao is. Classical Taoist texts such as the I Ching and the Tao Te Ching characterize the Tao as unnamable, incapable of being expressed in language. The Tao has thus typically been linked to negative concepts such as emptiness, limitlessness, or nothingness (Ozaki 2001, Chia and Wei 2009, Grigg 1994). If the Tao is indeed associated with nothingness, and if Taoism forms the foundation of the Pythagorean Lambdoma as Haase suggests, we might expect the zero-dimensional interval of the Lambdoma, $0 / 0$, to reflect nothingness. In confirming this, Godwin first underscores the special status of $0 / 0$ as that which "sounds no tone but is the silence toward which all tones tend" $(1992,192)$. Then, working with the analogy between silence and Non-Being, Godwin suggests that, just as the positive tones are rooted in the silence of $0 / 0$, "all Being culminates in a Nothingness beyond Being that ... is paradoxically its sole support and positive origin" (193). We may thus take $0 / 0$ as the Pythagorean expression of the Tao.

Taoist thinkers, drawing from the earlier Yin-Yang School of philosophy (the School of Naturalists), posited the idea that, from the nothingness of the Tao there arises the paradoxical duality of yin and yang, the opposed yet interpenetrating forces that drive the dynamics of nature. But the I Ching goes further, identifying two additional levels of evolution from the original Tao. As theologian Whalen Lai puts it: "The I Ching says: From the Great Ultimate [i.e., the Tao] come the two poles [yin and yang]; from the two poles come the four forms [... and] from the four forms [bigrams] come the eight trigrams" $(1980,254)$. Lai provides a diagram of this (Fig. 13).

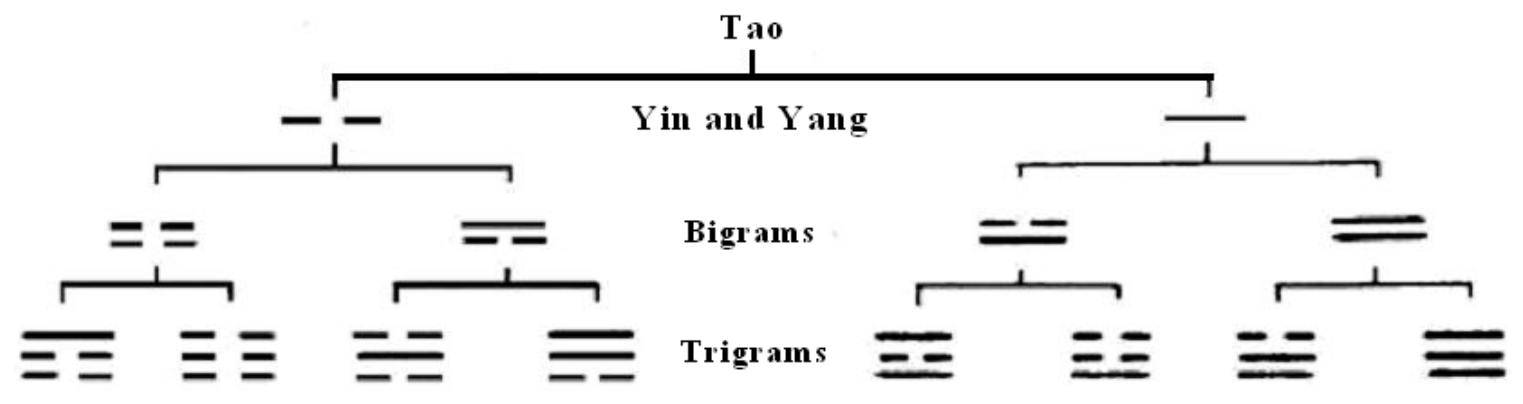

Figure 13. The yin-yang "family tree": four levels of cosmogony (adapted from Lai 1980, 254). Copyright (C) 2008 by John Wiley \& Sons, Inc. Reprinted by permission of John Wiley \& Sons, Inc.

Having considered above the proposition that the values of the topodimensional action matrix (Table 1) are correlated with the values of the Pythagorean table (Table 2), I would now like to extend this to include the four members of the yin-yang family depicted in Figure 13. We have already seen the involvement of two family members: (1) the Tao, associated with the $0 / 0$ 
Pythagorean interval and the zero-dimensional, sub-lemniscatory member of the topodimensional family; and (2) the trigrams, linked to the 3/3 Pythagorean interval and the three-dimensional, Kleinian member of the topodimensional family. In due course, we will see how the other two branches of the yin-yang family-the unigram mode of yin and yang and the bigrams - are correlated with the one- and two-dimensional members of the topodimensional family, respectively. For the moment, I will just observe that the numbers of yin-yang lines correspond directly to our topodimensional numbers. The Tao itself is devoid of yin-yang lines and this matches the zero- dimensional term of Table $1, \varepsilon_{\mathrm{D} 0}$. The single yin-yang lines emerging from the Tao parallel the one-dimensional term, $\varepsilon_{\mathrm{D} 1}$. The yin-yang counterparts of the two-dimensional lifeworld $\left(\varepsilon_{\mathrm{D} 2}\right)$ are the double lines constituting the bigrams, and the triple lines comprising the trigrams relate to the threedimensional realm $\left(\varepsilon_{\mathrm{D} 3}\right)$. Evidently then, the number of yin-yang lines bears a simple, one-to-one relationship to dimension number.

Now, let us focus again on the trigrams. In the first two sections of this paper, we investigated the I Ching's Ho-t'u number archetype and found that its associated trigrams are organized into two cycles of four phases each, with phase action following the underlying topology of the Klein bottle. The cosmogonic implications of this were explored and we were led to the provisional conclusion that cycle 1 involves the generation of forward or clockwise action entailing an expansion of the universe that projects the appearance of an objective physical world. Then, in Kleinian cycle 2 , the gears shift to backward and the projection is withdrawn, the universe contracting to bring to awareness the psychophysical nature of reality. What we have come to realize in subsequent sections is that the Klein bottle and the Ho-t'u, besides being related to one another, are each members of whole families of interrelated structures - one family topodimensional, the other Taoist. For a full understanding of cosmogony, the entire family must be taken into account in each case, so we clearly will need to work out the relationships among all the members of both cosmic families. Moreover, while the overview of dimensional generation offered in section 4 provides a general idea of the pattern of cosmic evolution giving rise to the fundamental forces of nature, it accounts only for the expansion of the universe, not its subsequent contraction as adumbrated in the Kleinian Ho-t'u. The aspect of contraction surely must be included.

In the next section, I offer a general interpretation of dimensional development that addresses the issue of lifeworld expansion and contraction. Then, in section 7, the specific stages of cosmogony are laid out for the topodimensional family, and this is followed by a section showing that the family of Tao lends itself to essentially the same analysis. What comes through in these sections is the deep affinity and underlying harmony of Western and Eastern "string theories."

\section{GENERAL STAGES OF DIMENSIONAL DEVELOPMENT}

Phenomenological thinking provides us with an insight into the embodied dialectical interplay of object, space, and subject lying behind the Cartesian facade of a changeless space wherein objects are cast before detached subjects.

Phenomenologically, space is not a static context for the mechanistic transformation 
of externally related objects; rather, the lifeworld dimension is a "spatio-subobjective" being (what Merleau-Ponty called "an 'element' of Being"; 1968, 139) that transforms itself organically. We can see from this that the phenomenological approach lends itself to the idea of dimensional generation in a way that classical thinking does not. The lifeworld dimension does not just contain earthly matter but is itself of the earth. Like earthly matter, this dimension is in process. And we are about to see that, like living matter, it passes through stages of biogenesis. Thus the phenomenological dimension possesses the character of a living organism-though not a finite particular organism, to be sure. Instead the dimension in question is a generic organicity, a whole dimension of life. And that dimension develops.

Dimensional development deals not with changes taking place in an individual or subject per se, but with how the very relationship of subject, object, and space changes. In the The Self-Evolving Cosmos we discover that the Cartesian framework of object-in-space-before-subject that seems to preclude an organismic basis for dimensional change in fact itself arises in the intermediary stage of a developmental process that is indeed organically dimensional. In the opening stage, object, space, and subject are largely undifferentiated. Rather than constituting welldefined ontological categories, they comprise only an incipient flux of embryonic possibilities. In the earliest fragment of Western philosophy, Anaximander referred to this inchoate condition as the apeiron (see Rosen 2004). Literally meaning "without measure," the old Greek word was variously interpreted as "limitless," "boundless," "indeterminate," or "unintelligible" (Angeles 1981, 14). In the protoscientific discipline of alchemy, the initial state of affairs was termed prime matter: "prima materia, which is the original chaos and the sea" (Jung 1970, 9). Then, from the primordial flux, the first differentiation of subject and object emerges within the medium of a nascently differentiated space. This marks the beginning of stage 2 and, as it unfolds, distinctions among subject, object, and the space that contains them harden into categorical divisions that are now assumed to have been there from the start. Completely overshadowed is the dialectical process that gave rise to these divisions. In thus expanding from the "black hole" of the primeval lifeworld, an extensive universe is opened up and the original connection with the psyche is obscured. What began as a dense psychophysical "soup" is now projected as a purely physical world stretching "out there" before us, an objective realm from which the lived subject has been dropped. (Earlier I gave an example of this objective stance in discussing the posture of conventional mathematics wherein attention is always focused on the mathematical object, with the subjectivity of the mathematician seldom being acknowledged.)

The first two general stages of dimensional transformation have their counterparts in the Kleinian action of the Ho-t'u described in section 2. The initial stage corresponds to the original "counterclockwise" action from which clockwise action is generated in Ho-t'u cycle 1. Since the paper model of the Klein bottle giving the topology of the Ho-t' $u$ is itself but an object in three-dimensional space, it cannot show that the opening phase of cycle 1 actually entails neither clockwise nor counterclockwise action, for this undifferentiated state of affairs affords no definite orientation. The three subsequent phases of Ho-t'u cycle 1 parallel the second general stage of dimensional development: "the usual clockwise movement, 
cumulative and expanding as time goes on." It is here that an objective world is projected and time flows forward in its ordinary sequence according to time's arrow of increasing entropy in an expanding physical universe.

What of the third general stage of dimensional development? This coincides with the second cycle of the Kleinian Ho-t'u. We have already considered the proposition that, in entering cycle 2 , the gears shift from clockwise to counterclockwise, forward to backward, and the universe contracts. As this process is enacted, the projection of a purely physical three-dimensional universe is withdrawn. It is by overcoming our fixation on the external world of objects and drawing our attention inward that we become aware of the psychophysical nature of the cosmos. We are going to see that, in thus moving backward, lower-dimensional lifeworlds concealed in earlier stages of cosmogony are brought to light. The I Ching implies that, through the cosmic contraction and reversal of time that takes place in cycle 2 , "the seeds of the future take form." In this regard, Wilhelm says in the $I$ Ching commentary I cited section 1: "If we understand how a tree is contracted into a seed, we understand the future unfolding of the seed into a tree" (Wilhelm quoted in von Franz 1974, 236-37). I will shed light on the meaning of this enigmatic passage in the next section.

\section{THE COSMOGONIC SPIRAL}

The description of dimensional development just given does not take into account the fact that each member of our topodimensional family has its own distinctive pattern of change, its own unique phase structure. We have learned that dimensional transformation generally entails stages of expansion and contraction, forward-directed stages in which a world is projected and retrograde stages in which the projection is withdrawn. But we have not yet seen how this is specifically orchestrated for the four fundamental lifeworld "tones" that sound the music of the dimensional spheres. So the time has come to bring our picture of cosmogony into sharper focus.

Consider again Table 1, the interrelational matrix of topodimensional spin structures. Taken by itself, this table affords but a static picture of dimensional associations, one that is "averaged over," i.e. abstracted from, the actual facts of dimensional change. Therefore, to fill in the concrete details of how the several dimensional spinors evolve in relation to one another, we must set the matrix in motion. This is achieved in Table 3. 


\section{Clockwise Stages Counterclockwise Stages}

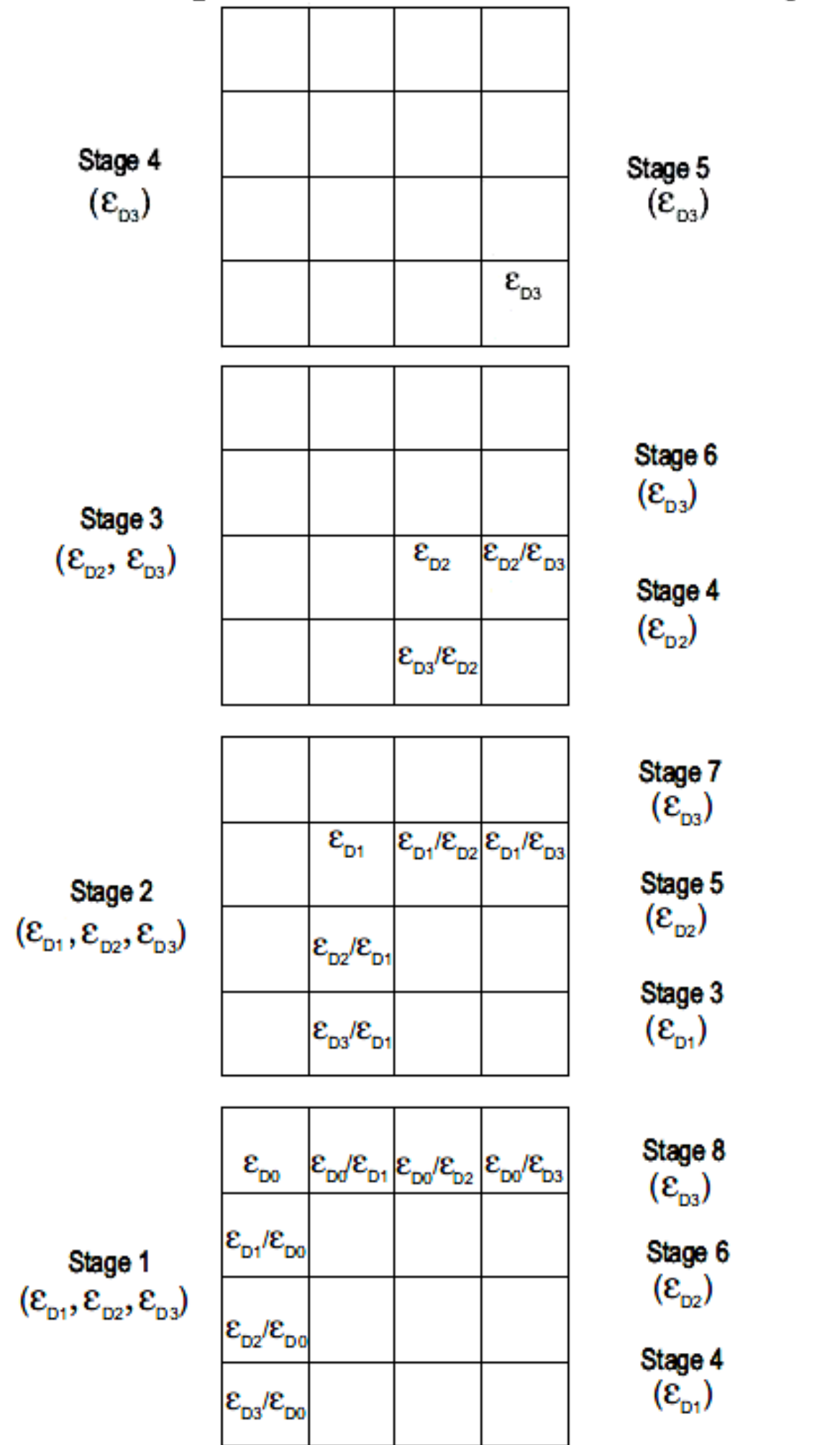

Table 3a. Stages of Dimensional Generation 
Table 3a displays the full course of development of all orders of topodimensional action. In effect, the Table expands Table 1 diachronically, now showing the stages of contraction as well as those of expansion. While the hypernumber values given in the cells of these matrices are the same as those exhibited in Table 1, with the new table we can study the specific lines of evolution of the spin structures that the hypernumbers represent. Employing the principle of synsymmetry set forth in section 4 , we will track these interwoven cosmogonic pathways.

Generally speaking, each matrix of Table 3a features on its main diagonal (that arrayed from upper left to lower right) one of our four fundamental spinors. These principal terms $-\varepsilon_{\mathrm{D} 0}, \varepsilon_{\mathrm{D} 1}, \varepsilon_{\mathrm{D} 2}$, and $\varepsilon_{\mathrm{D} 3}$-correspond to the four reflexive selfdivisions $(0 / 0,1 / 1,2 / 2,3 / 3)$ constituting the series of fundamental tones of the Pythagorean table (Table 2), which, in turn, are aligned with the topological series of self-intersecting forms (Fig. 12). The hypernumber ratios appearing off the main diagonal of each matrix are members of enantiomorphic pairings that pertain to developmental relationships between the different dimensional spinors (as will soon be discussed).

Table 3a is basically to be read in a circular fashion: we begin by reading it from bottom to top, then reverse course to read it from the top down. Starting from the matrix at the bottom, the upward movement through the four matrices gives the clockwise, forward, projective stages found in cycle 1 of dimensional generation, indexed by the stage values appearing to the left of the matrices. We then switch to the counterclockwise or retrograde stages of cycle 2 wherein projections are withdrawn, now reading back down through those same matrices, with stage numbers presently displayed to the right. The parenthetic hypernumber terms accompanying each stage number indicate the topodimensional spinor or spinors to which that stage number applies; since the zero-dimensional spinor does not undergo development, it does not appear here. If the sequence of stages for each topodimensional spinor is considered separately from that of the other spinors, we see that Table 3a in fact does not describe the action of a single circle (upward through the clockwise stages, downward through the counterclockwise ones) but of circles nested within circles, so that the overall pattern is actually that of a spiral.

To preserve the thoroughly interwoven, nonlinear character of dimensional interrelatedness, Table 3a displays the several windings of the dimensional spiral as overlapping one another. However, this makes the Table somewhat difficult to read. To facilitate understanding, I offer Table $3 \mathrm{~b}$ as a visual aid. Here the circulations of the dimensional spiral have been parsed, teased apart for easier identification. 
$\varepsilon_{\mathrm{D} 0}$

Sub-Lem niscatory Winding

Cycle 1
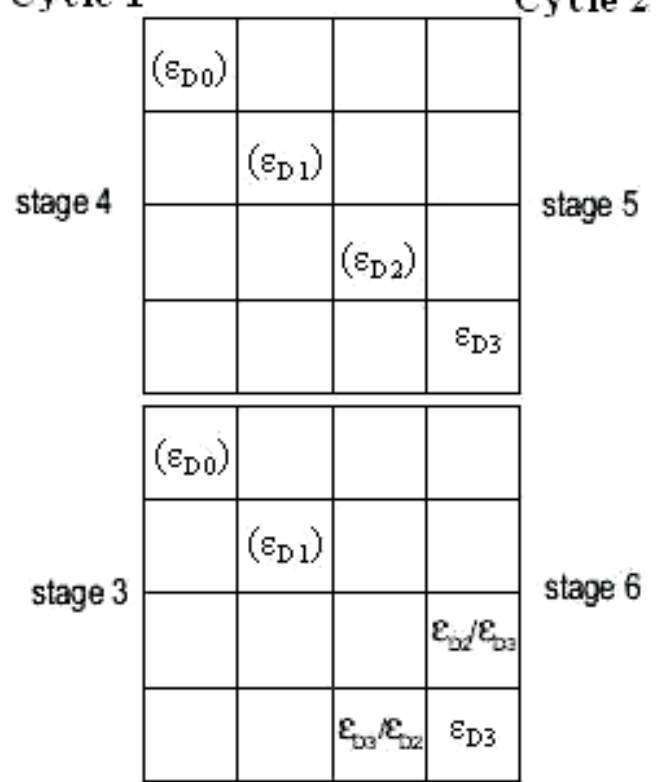

\begin{tabular}{|l|l|l|l|}
\hline$\left(\varepsilon_{\mathrm{D} 0}\right)$ & & & \\
\hline \multirow{2}{*}{ stage 2 } & & & $\varepsilon_{\mathrm{O} 1} / \varepsilon_{\mathrm{L} 3}$ \\
\hline & & & \\
\hline & $\varepsilon_{\mathrm{D} 3} / \varepsilon_{\mathrm{D} 1}$ & & $\varepsilon_{\mathrm{D} 3}$ \\
\hline
\end{tabular}

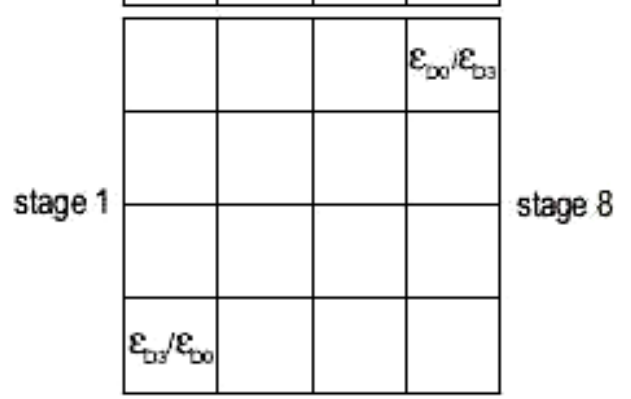

Kleinian Winding

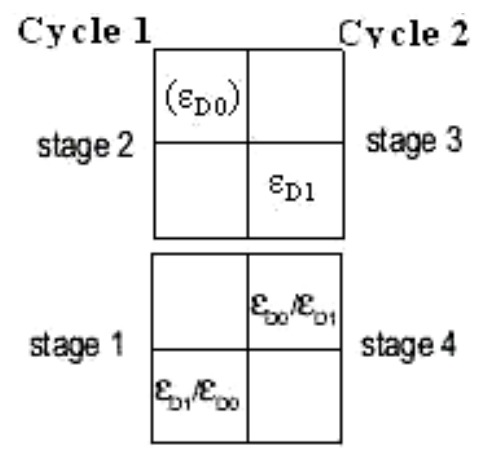

\section{Lem $\mathrm{n}$ is catory $\mathrm{W}$ inding}

Cycle 1
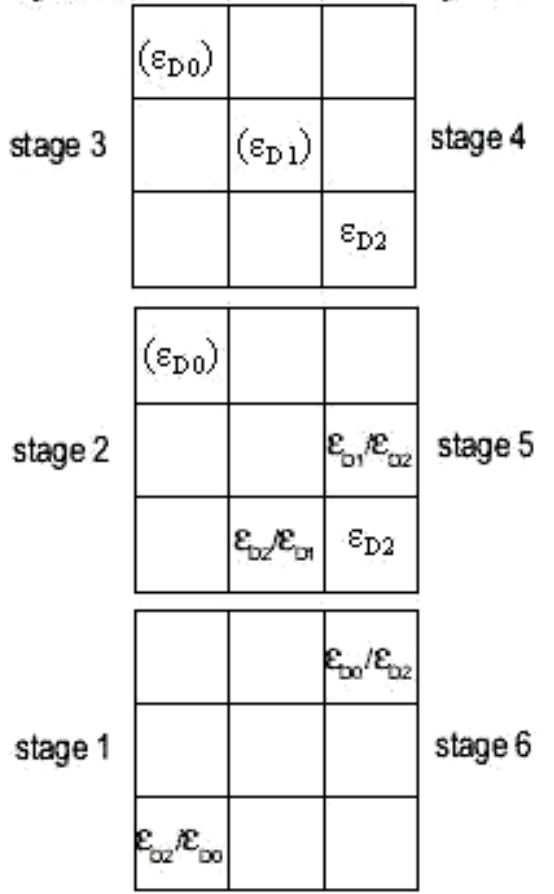

Mo ebial Winding
Table 3b. Cosmogonic spiral, parsed into separate windings 
The driving force behind the generation of lifeworld dimensions depicted in Table 3 was already identified in section 4: an $n$-dimensional lifeworld is crystallized through the fusion of $n$-1-dimensional enantiomorphs. Involved here is the dialectical interplay of symmetry and asymmetry that I have termed synsymmetry. Prior to their fusion, enantiomorphs are non-identical mirror opposites, thus asymmetric with respect to each other. This condition of enantiomorphic asymmetry prevails at the beginning of a winding. Then, as cycle 1 of the winding progresses, the merger of $n$-1-dimensional enantiomorphs gives rise to $n$-dimensional symmetry (an example from section 4 is the fusion of opposing sides of the lemniscate to yield the Moebius strip). The synsymmetry concept further implies that the establishment of symmetry must be followed by a new order of asymmetry. This is initiated in cycle 2 of the winding. Here enantiomorphs are incubated for the $n$-dimensional structure (the Moebius strip, for example) that meet the necessary conditions for opening up the next winding, that wherein $n$ dimensional enantiomorphs will fuse to generate $n+1$-dimensional symmetry (e.g., the symmetry of the Klein bottle). It is this synsymmetric process of dimensional fusion and "diffusion" (i.e., the creation of asymmetric enantiomorphs) that fuels the movement from one winding of the cosmogonic spiral to another. I suggest, moreover, that the synsymmetry idea helps us comprehend the meaning of the $I$ Ching passage cited earlier: "If we understand how a tree is contracted into a seed, we understand the future unfolding of the seed into a tree." In the cosmic contraction and time reversal occurring in cycle 2 of a given winding, "the seeds of the future take form" by propagating the enantiomorphs required for generation of new symmetry (a new "tree") in the next winding.

In Table 3b, we see the progressive increase in the number of stages through which dimensional development occurs as we go from lower- to higher-dimensional windings of the dimensional spiral via the process of synsymmetry. The first winding of the spiral is that of the sub-lemniscatory matrix, which consists of but a single cell. In effect, the "radius" of this circulation is "zero," for it entails no transformation whatsoever. ${ }^{8}$ No stages of development can be found for $\varepsilon_{\text {Do }}$ nor are any necessary, since this zero-dimensional sphere is comprised only of the seeds of higher-dimensional structures whose generation will be facilitated in subsequent windings by the support they receive through $\varepsilon_{D}$ 's enantiomorphic overtones $\left(\varepsilon_{\mathrm{D} 1} / \varepsilon_{\mathrm{D} 0}, \varepsilon_{\mathrm{D} 2} / \varepsilon_{\mathrm{D} 0}, \varepsilon_{\mathrm{D} 3} / \varepsilon_{\mathrm{D} 0}\right)$ and undertones $\left(\varepsilon_{\mathrm{D} 0} / \varepsilon_{\mathrm{D} 1}, \varepsilon_{\mathrm{D} 0} / \varepsilon_{\mathrm{D} 2}, \varepsilon_{\mathrm{D} 0} / \varepsilon_{\mathrm{D} 3}\right)$.

Advancing to the second winding of the spiral, the matrix expands to the $2 \times$ 2 structure associated with the generation of the one-dimensional lemniscatory lifeworld. The opening stage finds a primordial matrix in which the one-dimensional spinor resides in but an embryonic form. In our psychophysical string theory, where spinning particles can be seen as vibrating strings creating waves that possess

\footnotetext{
8 Of course, the "radii" of all dimensional circulations are "zero" in the sense that stage transitions are not actually displacements in an extensive spatial continuum, though they appear as such in Table 3. The several dimensional circulations portrayed in the Table cannot really have finite radii becauseinstead of taking place within space, they constitute the quantized pre-spatial actions from which space first arises.
} 
overtones and undertones, we may characterize the zero-dimensional sublemniscatory spinor as a "carrier wave" whose $\varepsilon_{\mathrm{D} 1} / \varepsilon_{\mathrm{D} 0}$ overtone and $\varepsilon_{\mathrm{D} 0} / \varepsilon_{\mathrm{D} 1}$ undertone carry the fledgling one-dimensional spinor (see "Waves Carrying Waves," chapter 7 of Cosmos). One-dimensional action is but nascently oriented here; the differentiation of the lemniscatory lifeworld has not yet taken place.

Moving upward now to the stage- 2 matrix of the lemniscatory winding, we see that $\varepsilon_{\mathrm{D} 1}$ has gained maturity. This entails the fusion of the asymmetric sublemniscatory enantiomorphs, $\varepsilon_{\mathrm{D} 1} / \varepsilon_{\mathrm{D} 0}$ and $\varepsilon_{\mathrm{D} 0} / \varepsilon_{\mathrm{D} 1}$, wherein they are "annihilated," being absorbed into the emergent symmetric structure of the expanding lemniscatory universe (to be more exact, $\varepsilon_{\mathrm{D} 1}$ is the mature quantized spinor that projects an expanded universe). However, with the transition to stage 2 , the primordial state of affairs is not simply left behind; rather, it is relegated to the background. And while the primal potency of $\varepsilon_{\mathrm{D} 0}$ is in eclipse, a depotentiated, objectified version of it is projected as a point-like lower dimension in the onedimensional world (concrete examples of this will be given in section 9 when we are working with particle evolution). The attenuation of $\varepsilon_{\mathrm{D} 0}$ is represented in Table $3 \mathrm{~b}$ by placing this value in parentheses. At the same time that the sub-lemniscate is projected as a zero-dimensional object in the one-dimensional world, the lemniscatory world is itself projected as something objectively "out there." So forward-oriented projective activity is occurring in earnest in this second stage of cycle 1 . Whereas the lemniscate's action is but incipiently oriented in stage 1 , it presently assumes a definite clockwise direction.

The completion of stage 2 brings the first cycle of lemniscatory archetypal action to a close. A critical turning point is now reached where the gears shift from forward to backward, clockwise to counterclockwise. Entering the second cycle, the expansion of the one-dimensional world is reversed and its projection is withdrawn. ${ }^{9}$

Let us look more closely at the nature of this act of withdrawal, or what we may call retrojection. It does not imply that the forward movement of cycle 1 simply ceases in cycle 2 . Projective action continues, and yet it is transformed by being counter-acted. What exactly does this mean? Suppose you were handling a textured piece of fabric, one whose fibers were arranged in a certain direction. Is it not by running your fingers against the grain of the material that its direction becomes more clearly discernable? Similarly, in the cycle-two backward movement against the grain of cycle one, awareness is gained of the very process of forward-directed projection that transpires in that first cycle. It is in this way that the projection is retracted, consciously taken back, in the midst of its ongoing occurrence.

The retrograde orientation is established in stage 3 of the lemniscatory winding. What was projected as an objective, "purely physical" one-dimensional

\footnotetext{
${ }^{9}$ In the interest of manageable exposition, action cycles are not shown topologically but appear as linear sequences of matrix cells in Table 3 . Thus, in the lemniscatory winding of Table $3 b$, cycle 1 is comprised of stages 1 and 2, and cycle 2 consists of stages 3 and 4 . But these phases of action are indeed topological and a more accurate way of indicating the double cyclicity of the lemniscate would be via the emblem of infinity that tangibly displays the lemniscate's two cycles of opposing clock orientations: $\infty$.
} 
world in stage 2 is presently recognized as first arising from the symmetry-creating psychophysical act of fusing the sub-lemniscatory enantiomorphs of stage 1 . This basically accords with von Franz's assessment of the cycles of the Ho-t'u, where she identifies Ho-t'u cycle 1 as "physical" and cycle 2 as "psychic" (see section 1). It should be clear however, that the one-dimensional realm of the lemniscate is far simpler than the three-dimensional world of the Kleinian Ho-t'u that von Franz was dealing with. The greater number of stages in the Kleinian winding shown in Table $3 \mathrm{~b}$ reflects the fact that development goes further here, with a sharper division emerging between subject and object, psyche and matter. We can say in general that the progressively increasing number of stages found in passing from one winding of the cosmogonic spiral to another indicates the growing complexity of the lifeworlds generated.

Note that in stage 3 of the lemniscatory winding, the awareness gained of the stage-1 source of projection is somewhat limited inasmuch as this cognizance of the zero-dimensional realm itself remains strictly one-dimensional. It is lemniscatory consciousness that prevails in stage 3 as it did in stage 2 and the lemniscate's abstract perception of the sub-lemniscatory sphere does not return it to that primeval setting in a concrete way. Although the projection of the one-dimensional world has been effectively withdrawn in stage 3 , that of the zero-dimensional world has not (in the Table, $\varepsilon_{D 0}$ remains enclosed in parentheses, indicating its continuing presence as a depotentiated projection).

It is through the retrojection occurring in stage 4 that the repression of the sub-lemniscate is lifted and its potency restored. Here, rather than merely apprehending the sub-lemniscate from a lemniscatory perspective, the lemniscate is carried back to its embryonic origin and the zero-dimensional structure itself gains full presence. This return to the beginning is no mere regression in which the mature lemniscate utterly reverts to its incipient form. Despite Table $3 \mathrm{~b}$ 's linear sequencing of stages, they are in fact not simply separated from each other but overlap. Therefore, just as the zero-dimensional structure of stage 1 is not left behind in passing to stage 2 but continues in the background, the mature onedimensional structure of stage 3 maintains its presence in stage $4 .{ }^{10}$

With the retrojection enacted in stage 4 , closure is brought to the lemniscatory winding of the cosmogonic spiral. The fully developed lemniscate and revitalized sub-lemniscate are now both on the scene. They are wholly present to each other and can enter into resonance to sound the "music of the dimensional spheres." Because the relationship is no longer that of a mature spinor carrying a spinor that is still undeveloped, because both dimensional spinors are now mature, we can reinterpret the enantiomorphic ratios as expressing the intimate reciprocity of archetypal players.

The second cycle of the lemniscatory winding not only completes the development of the lemniscate but also paves the way for entry into the Moebius winding. For it is here, by the synsymmetric process of dimensional diffusion, that

\footnotetext{
${ }^{10}$ It is true, however, that while stages overlap one another in both cycles of dimensional development, the quality of overlap in cycle 2 differs from that in cycle 1 . See Cosmos, Chapter 10, for a discussion of "transparent" and "translucent" forms of stage overlap.
} 
the lemniscatory "tree" contracts into a "seed" required for the growth of the Moebius "tree." The other "seed" necessary for subsequent Moebius development is of course the de-repressed sub-lemniscate. The seeds synchronously sown in cycle 2 of the lemniscatory winding become the asymmetric carrier waves whose enantiomorphic fusions generate Moebius symmetry in the next topodimensional epoch.

When the lemniscatory winding closes, the cosmogonic spiral opens out into the Moebius, consisting of $3 \times 3$ matrices transformed over six stages. The compass has shifted so that initial action is once again unoriented. In stage 1 of this twodimensional circulation, we find that-as a result of the germinal activity in the second cycle of the last epoch-the carrying capacity of the zero-dimensional sublemniscate has been enlarged. Through its new pair of enantiomorphs, $\varepsilon_{\mathrm{D} 2} / \varepsilon_{\mathrm{D} 0}$ and $\varepsilon_{\mathrm{D} 0} / \varepsilon_{\mathrm{D} 2}$, the sub-lemniscate serves as carrier wave for the embryonic Moebius spinor. The fusion of these asymmetric enantiomorphs on behalf of the developing Moebius then brings us into stage 2 . With the sub-lemniscatory carrier having been absorbed into the Moebius wave, the original zero-dimensional spinor is eclipsed and an attenuated version of it is projected (as indicated by the appearance of $\varepsilon_{\mathrm{D} 0}$ in parentheses). For its part, the Moebius gains its measure of symmetry, becoming more mature. A clockwise orientation is assumed in this stage, and we have the first projection of an objective two-dimensional world stretching before an emergent two-dimensional subjectivity. However, whereas the one-dimensional lemniscate required the fusion of but a single pair of enantiomorphs to complete its cycle-1 development, the more complex Moebius pattern calls for two such fusions.

This is where the lemniscatory contraction of the previous winding bears fruit. Its effect is that the lemniscate can currently play the role of a carrier wave, expressed as the enantiomorphic overtone-undertone coupling, $\varepsilon_{\mathrm{D} 2} / \varepsilon_{\mathrm{D} 1}$ and $\varepsilon_{\mathrm{D} 1} / \varepsilon_{\mathrm{D} 2}$. It is the fusion of these enantiomorphs that brings us into the third stage of Moebius cosmogony, the conclusive stage in its forward-directed unfoldment. With the merger of lemniscatory enantiomorphs, the lemniscatory carrier wave dissolves into $\varepsilon_{\mathrm{D} 2}$ and a depotentiated form of it is projected (denoted in stage 3 by its enclosure in brackets). This attenuating projection is accompanied by a second and final projective expansion of the two-dimensional world. The now-mature Moebius spinor thus reaches full symmetry.

Next comes the transition from cycle 1 to cycle 2 of the Moebius winding. Entering stage 4, the gears are put into reverse, expansion switches to contraction, and two-dimensional spin is reoriented, becoming counterclockwise. In this first retrograde movement, there is an initial recognition that the cycle- 1 preoccupation with an objective physical reality external to the perceiving subject has actually arisen from $s u b$-objective, psychophysical acts of projection. (Do note that subjectobject relations in the Moebius winding are simpler, less differentiated, than those in the Kleinian winding; see Rosen 2006 and 2008a for an intensive examination of this distinction.) But the stage-4 withdrawal of the two-dimensional projection leaves untouched the depotentiating lower-dimensional projections. Then, moving concretely backward in stage 5 , two-dimensional retrojection is taken further. The earlier (stage-2) projection of $\varepsilon_{\mathrm{D} 2}$ is counteracted and the repression of the onedimensional lemniscatory carrier wave is lifted. The Moebius spinor now enters into 
harmony with its lemniscatory counterpart, and we interpret the enantiomorphic ratios of stage 5 as expressing that harmony.

Going still further back in stage 6, the zero-dimensional sub-lemniscatory carrier emerges from obscurity to become harmonically attuned to the Moebius. The projective actions of cycle 1 have now been wholly counteracted and the Moebius spinor has achieved synchrony with its lower-dimensional relatives in the topodimensional family. And since the synchrony is realized amidst the process of contraction, these three spinors come to constitute the seed-structures manifested as carrier waves in the next epoch.

With the completion of the Moebius winding, the dimensional spiral dilates once again and we find ourselves in the opening stage of the Kleinian winding of $4 \times$ 4 matrices evolving over eight stages. Beyond its incipiently oriented stage, the three-dimensional Kleinian organism gains symmetry and matures through three clockwise phases. This follows the pattern of the earlier windings. In each stage a different fusion of enantiomorphs occurs entailing the absorption of a lowerdimensional carrier wave (sub-lemniscatory, lemniscatory, and Moebial) that facilitates the growth of the Kleinian wave. And with each new fusion in the cycle-1 expansion of Kleinian dimensionality, there is a new and more differentiated projection of an objective three-dimensional world.

Subsequently, the gears are reversed once more and we pass into the second cycle of Kleinian evolution, where expansion becomes contraction and clockwise action becomes counterclockwise. Stage 5 brings the Kleinian organism's first realization that the "objective physical universe" is in fact a psychophysical projection. In the three ensuing stages of cycle 2, the cycle-1 repressions imposed on the lower-dimensional carrier waves are successively lifted and each enters into resonance with the Kleinian dimension. With all the members of the topodimensional family resounding in harmony, the "symphony of dimensional spheres" is heard in its fullness.

But what happens next? Does the cosmogonic spiral now close into a circle that brings us to the end of cosmogony? What of the logic of synsymmetry, of dimensional fusion and diffusion? In cycle 2 of the Kleinian winding, must the Kleinian "tree" not contract into a "seed" for a new, meta-Kleinian "tree," a new round of topodimensional transformations in a novel winding of the spiral? This implication is inescapable if we are to avoid abandoning arbitrarily the synsymmetry principle so critical to our analysis. Indeed, when the analysis was related to the Pythagorean table in section 5, I noted that this table is usually portrayed as an indefinitely expanding series of musical intervals that goes beyond the 3/3 interval correlated with Kleinian three-dimensionality. Nevertheless, with the prospect of surpassing the Kleinian dimension, it appears we have reached a watershed, for our analysis of cosmic evolution has come up against the limits of our own analytical capability. While we can abstractly anticipate higher-dimensional windings of the cosmogonic spiral, we cannot gain concrete knowledge of them because they lie beyond the conceptual framework we employ as three-dimensional analysts.

I do not hold with the Kantian view that the tangible experience of a dimension transcending our cognitive frame is forever inaccessible to us. Instead I 
suggest that we can indeed come to palpably realize the higher dimension but that doing so requires more than just changing the content of our analysis. Our analytic framework itself must evolve, stretching to accommodate the new dimension. Before I am finished, I will attempt to clarify what this means and what it may require.

To sum up this section, the foregoing analysis brings to light the stages of dimensional development that serve to distinguish one topodimensional spin structure from another. While the several windings of the dimensional spiral overlap, each lifeworld circulation runs its own course, with distinct circulations being marked by differences in the number of stages that each requires to carry out its clockwise projections and counterclockwise retrojections. These differences reflect, in turn, differing degrees of lifeworld complexity, which show up in the capacity for discriminating subject and object, psyche and matter.

\section{THE COSMOGONIC SPIRAL IN LIGHT OF THE TAO}

In completing our study of how dimensions evolve, we have reached another crossroad between West and East. For we are now prepared to grasp the relationship between our section-7 treatment of the Klein bottle in the context of dimensional generation and our approach to it in section 2, where it is taken in conjunction with Taoism's Ho-t'u and associated trigrams.

In the earlier section, the quantized phase structure of the Klein bottle was seen to correspond to that of the Ho-t'u: both consist of two oppositely directed cycles of four phases each. This is of course the same structure manifested in the Kleinian winding of the cosmogonic spiral. What we could not see in section 2 is the thoroughly interrelational nature of Kleinian development. This only comes to light when we take into account the Klein bottle's membership in its topological family. Table 3 shows how each stage in the unfoldment of the Klein bottle is defined by its relationship to another family member. And what we see in Table 4 is not only the correspondence of the Kleinian winding of the cosmogonic spiral to the trigrams of the Ho-t'u, but the correlation of all members of the topodimensional family with the family of Tao. 
Winding of the Tao:

0

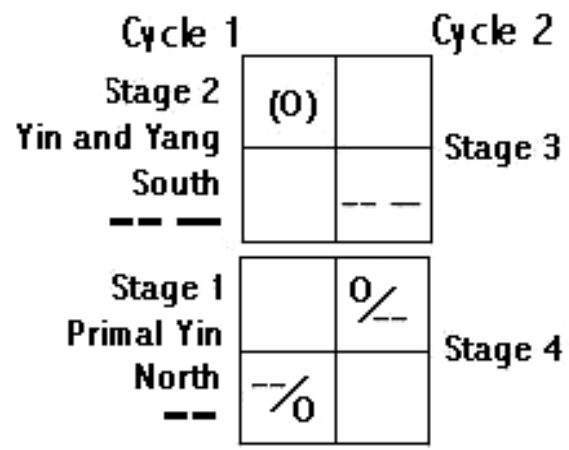

Unigram Winding
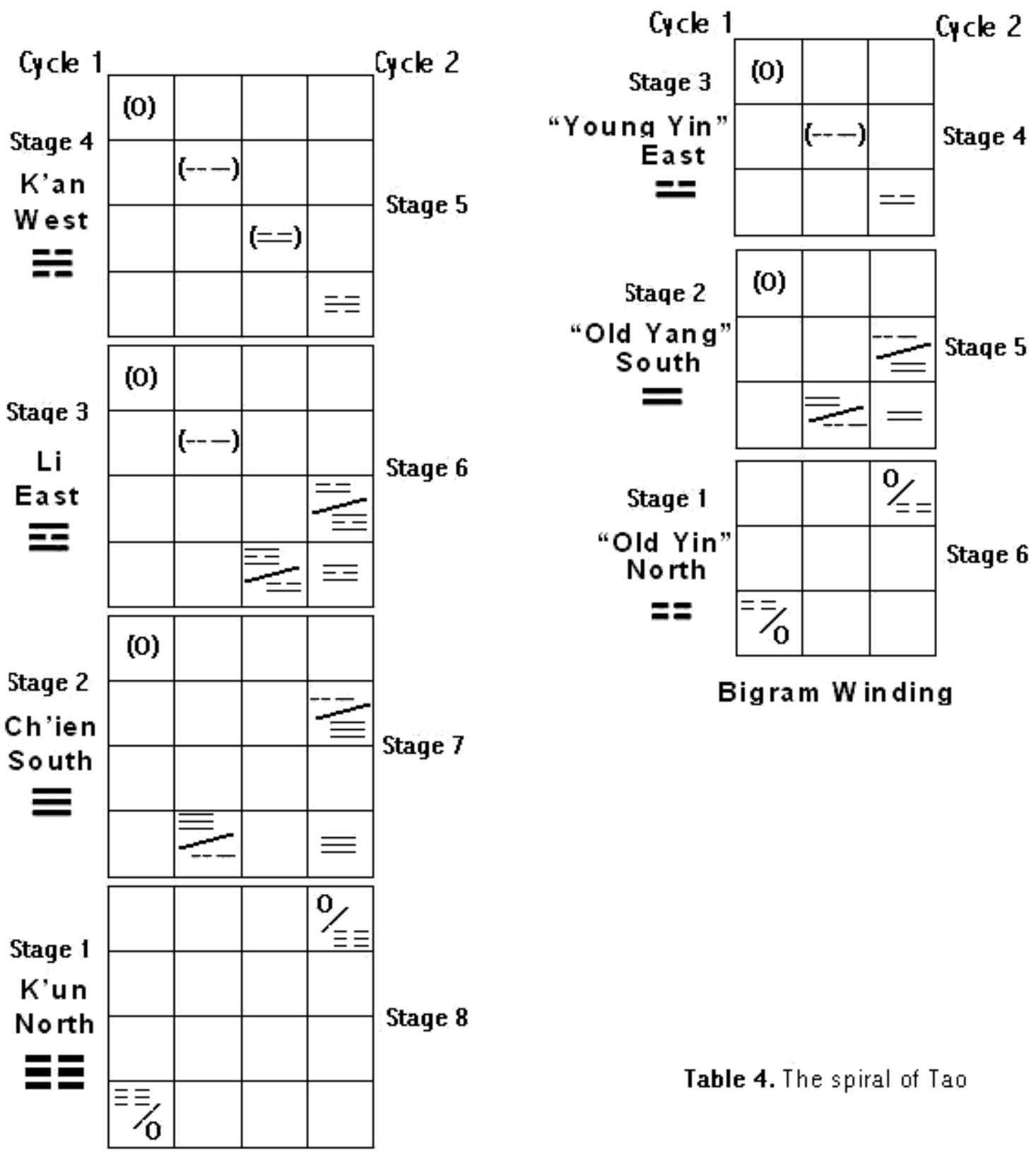

Bigram $W$ in ding

Trigram Winding

Table 4. The spiral of Tao 
For Table 4, I have replaced the topodimensional values of Table $3 \mathrm{~b}$ with their intimately related Taoist counterparts. The four orders of the Tao are given in the four windings of the cosmogonic spiral. The first winding consists of the Tao itself, shown in the upper left-hand quadrant of the Table as the single value, 0 . Whereas all other windings comprise extended developmental matrices, the Tao stands alone. As was said of the zero-dimensional sub-lemniscate, the Tao per se does not evolve through stages. Acting via its seed structures, it functions solely as a catalyst for the matrical generation of higher orders of itself: the unigrams, consisting of the basic modes of yin (--) and yang (-); the bigrams, entailing the doubling of the yin-yang lines; and the trigrams. Paralleling the generation of the topodimensional spinors, Taoist cosmogony is seen as driven by the synsymmetric process of enantiomorphic fusion and diffusion. Within each winding of Table 4, the unbracketed terms on the main diagonals of the matrices show the stages of development of the fundamental yin-yang spinors. The coupled enantiomorphs located off the main diagonals are the yin-yang ratio pairs, each of which expresses a relationship between members of two different orders of the yin-yang family. Cosmic evolution is facilitated by the symmetry-generating enantiomorphic fusion occurring in the first cycle of a given winding, followed by the cycle-2 diffusion that sows the seeds for the asymmetric enantiomorphs of the next winding.

Every winding of the Taoist cosmogonic spiral is to be read in the same circular manner as its topodimensional counterpart in Table 3. Reading through the stages of a given winding from the bottom matrix to the top, we have the clockwise, projective stages of cycle 1 . Then switching gears, we read downward from the top matrix, and this gives the counterclockwise stages of retrojection that constitute cycle 2 .

Let us begin our reading of Table 4 by reversing the sequence in which the windings were studied in Table $3 \mathrm{~b}$. We will not proceed from the Tao and track the cosmogonic spiral's expansion through the unigrams, to the bigrams and the trigrams. Instead we will start with the trigram winding since this is where we can best articulate a principle that will involve a significant departure from the classical treatment of the yin-yang family.

The trigram winding of Table 4 displays four $4 \times 4$ matrices evolving over two cycles of four stages each (à la the Kleinian winding of Table $3 \mathrm{~b}$ ). In the first cycle, we see the cosmogonic process by which the psychophysical trigram spinor progressively develops from an initially embryonic origin; as it matures and gains symmetry, it spins out an objective physical world. The trigram sequence follows that of the Ho-t'u cross. In the first stage, the embryonic spinor is represented by the trigram K'un, consisting of three broken lines; its compass direction is North. We observe in the bottom matrix of the winding the enantiomorphic support K'un receives from the Tao carrier wave (denoted by 0 ). Then, via the fusion of these enantiomorphs, we move upward to the next stage, where the spinor is now directed South and is manifested as Ch'ien, expressed by three solid lines. Not yet mature, Ch'ien is carried by the enantiomorphs of the mature yin-yang spinor originating in the unigram winding. Repeating the process of fusion, the trigram spinor advances to stage 3, assuming the structure of the Easterly-directed Li: a 
broken line between two solid lines. $L i$ is carried by the enantiomorphs of "Young Yin," the mature spinor of the bigram winding. (Bear in mind that a carrier wave always involves the mature form of a lower-order spinor, since a spinor cannot play the supportive role of carrier until its development is completed in its own winding.) The last projective stage of the trigram winding arises from the fusion of bigram enantiomorphs. In this phase, the trigram spinor reaches full maturity as $K^{\prime} a n$, a solid line between two broken lines. Note that the depotentiating projections previously discussed for Kleinian evolution are mirrored in trigram development: with each enantiomorphic fusion of cycle 1, the lower-order spinor that had supported the trigram's projective potentiation is itself subjected to a depotentiating projection that narrows it down and objectifies it, occluding its true nature. In Table 4, spinors thus attenuated are enclosed in brackets.

We know what happens in cycle 2. The gears shift from forward to backward, projection to retrojection, and the retrograde stages of the cycle unfold. The projections of cycle 1 are presently withdrawn, with lower-order spinors emerging from eclipse to enter into synchrony with the trigram spinor. And in the contraction of the trigram cosmos, these spinors become seeds for the next winding. Notice that, in Table 4, the stages of cycle 2 are associated with no new trigrams and accompanying compass directions, as they are in the classical rendition of the trigrams (see Figure 11). Why have I dropped four trigrams?

In the traditional approach to the I Ching, all eight permutations of the trigrams are employed and from this, 64 hexagrams are built for the purposes of personal guidance and divination, with philosophical and cosmological commentary added. What I am proposing is an alternative way of working with the trigrams that uses only the ones specified in cycle 1. Instead of adhering to the custom of introducing additional trigrams and compass directions for cycle 2, I suggest that the very same trigrams be employed, now in the retrograde orientation.

There is a sense in which the trigrams of cycle 1 and their affiliated compass points can be considered more fundamental than their cycle- 2 counterparts. First of all, we observe that the compass points conventionally featured in cycle 2 do not constitute directions that are uniquely different from the four cardinal directions arising in cycle 1 . The cycle- 2 directions are deemed "intercardinal" in that they combine the already established primary compass directions without introducing anything qualitatively new. We see from Figure 2 that the intercardinal directionsNorthwest, Southeast, Northeast, Southwest-are obtained simply by rotating the Ho-t'u cross by an angle of $45^{\circ}$ to give the compass markings that lie halfway between the cardinals.

Perhaps more importantly, while the classical trigrams of cycle $2-K e n, T u i$, Chen, and Sun-provide novel permutations of the triple yin-yang lines and these are subject to new interpretations, there is no change in the underlying combinatorial structure of the trigrams. The four trigrams of cycle 1 exhaust the possible combinations of broken and solid lines taken three at a time: all solid; all broken; two solid, one broken; two broken, one solid. What we have in the second cycle are but repetitions of those primary structures. The permutations are different but the more basic combinations stay the same. So, just as the classical compass points of cycle 2 provide no fundamentally new compass directions, the trigrams of 
this cycle offer no innovations in basic combinatorial structure. What happens when we limit the trigrams to the primary ones given in cycle 1 and take cycle 2 as a backward movement through those very same trigrams? We can then see clearly the isomorphic alignment of the trigram order of the yin-yang family with its Kleinian equivalent in the topodimensional family.

Moving backward now in the cosmogonic spiral of Table 4, let us consider the yin-yang winding that corresponds to the Moebius winding of Table $3 \mathrm{~b}$. The counterpart of the Moebius is the archetypal field that involves the generation of the bigram spin structure over two cycles of three stages each. In the classical literature, the set of bigrams is known as the Four Emblems or Four Symbols (Hulse 2002). The traditional bigrams are exhibited in Figure 13, along with their eight associated trigrams, only four of which we are employing in our combinatorial approach. Comparing Figure 13 with Table 4, we see the connection given in the former between the stage- 1 trigram $K^{\prime} u n$ with its three broken lines and the bigram composed of two broken lines, called "Old Yin" or "Greater Yin" (Hulse 2002, 379). Aligning bigram with trigram development, Old Yin is taken to signify the first stage of the bigrams. By the same token, the linking in Figure 13 of the stage- 2 trigram Ch'ien (three solid lines) with the "Old Yang" bigram composed of two solid lines suggests that Old Yang be associated with the second stage of the bigram winding. In Table 4, the third and final stage in cycle 1 of this winding corresponds to the bigram designated "Young Yin," consisting of a broken line atop a solid line. But what of the fourth bigram shown in Figure 13, made up of a solid line above a broken line? This bigram, named "Young Yang," is a permutation of the same combination of lines that constitutes Young Yin: a solid line and a broken line. In keeping with the principle of working only with basic combinations, one of these permutations must be excluded. Why has Young Yang been chosen for elimination and not Young Yin? Figure 13 shows that the former is connected with the stage-4 trigram K'an whereas the latter is linked to the stage-3 trigram Li. Since Table 4 maintains consistency in developmental sequencing by presenting the bigrams in the same stage order as the trigrams with which they are coupled, Young Yin must correspond to stage 3 of the bigram winding, leaving Young Yang as the extraneous permutation to be dropped. The compass directions for the three stages of bigram cycle 1 follow the sequence of the trigrams-North $\rightarrow$ South $\rightarrow$ East, with West excluded.

As with the trigram winding, that of the bigrams displayed in Table 4 shows the generation of the spinor through two cycles, the first expanding projectively to spin out an objective world (though one less differentiated and complex than the trigram world), the second contracting, moving backward, and withdrawing the projections of the first. Again we see the process of development facilitated in cycle 1 by mature lower-order spinors acting as carrier waves that give enantiomorphic support to the evolving spinor. In stage 1, Old Yin, the embryonic bigram, is supported by the enantiomorphs of the Tao. Enantiomorphic fusion then brings us to Old Yang in stage 2. Not yet fully developed, this bigram is carried by the enantiomorphs of the yin-yang unigram. Next, when unigram enantiomorphs fuse, we encounter Young Yin, the mature bigram spin structure of the third and final stage of projection. This is followed of course by the switching of gears. Passing 
backward through the bigram stages of cycle 1, the cycle- 2 spinor withdraws its projections and enters into harmony with the lower-order spinors that had been repressed on its behalf. In this contraction of the bigram universe, the spinors condense into germinating seeds, to bear fruit as the enantiomorphs that facilitate trigram generation.

We now go still further back on the cosmogonic spiral to the yin-yang equivalent of the lemniscatory winding. This lower-order archetypal field comprises a double cycle of spin transformations linked to the unigrams. Cycle 1 involves but two stages. In the first of these, we have the embryonic spin structure represented by a single broken line, designated "Primal Yin" (associated with the compass point, North). This spinor is carried by the enantiomorphs of the Tao. Just one fusion is needed to bring forth the mature structure of stage 2, denoted by single yin and yang lines (and oriented to the South). With the movement backward that ensues in cycle 2 , there is initial recognition in stage 3 that a world has been projected, followed by the more concrete retrojection enacted in stage 4 through which the Tao emerges from its stage- 2 eclipse to converge harmonically with yin and yang. The concomitant cosmic contraction forms the seeds for the bigram winding.

Let me emphasize the relative simplicity of the cosmogonic process in the unigram winding. As already noted, lower-order spin structures project less complex lifeworlds, with a lesser capacity for differentiating subject and object. We can say accordingly that the "objective physical world" projected in the unigram winding is but a weakly differentiated one involving a form of consciousness that is not as sharply focused and discriminating as the awareness achieved in higher dimensional windings.

Continuing back through the spiral to its source, we reach "that which inform[s] all the transformations in the cosmos" (Fowler 2005, 46): the Tao itself. The Tao, says Fowler, is what "generates the tension between opposites [i.e. yin and yang].... It is the quiet, spontaneous power that eternally gives energy to the cosmos, to the rhythmic composition of the stars and planets as much as to the energy that a tiny seed needs for germination" (46). The Tao is a primordial matrix. While this term is broadly defined in mathematics as an array of numbers, its general dictionary definition indicates an environment or material context in which something develops. Tao appears on its own in Table 4 as the single-celled matrix from which all develops. It is because the Tao is associated with silence, nothingness, and zero-dimensionality (see section 5) that we have assigned to it the value of 0 . Recalling Godwin's coupling of the Pythagorean $0 / 0$ with that which "sounds no tone but is the silence toward which all tones tend" $(1992,192)$, we now turn our attention back to the psychophysical "tones" of quantum gravity for a string theoretic specification of the "music of the dimensional spheres."

\section{THE SPIRAL OF QUANTUM GRAVITY}

In the past two sections, the evolution of the primary spinors was explained in highly theoretical terms. I would now like to flesh this out by showing its correspondence with the elementary forces of string theory. The Self-Evolving Cosmos describes in considerable detail the phenomenological approach to the 
standard model of particle physics. I offered a synoptic sketch of this in my 2015 article and must limit myself to an abbreviated rendition in the current paper as well. But the latter does go further than the former, and, in some important respects, the present analysis advances the work done in Cosmos itself.

What Cosmos suggests is that a full account of the fundamental particles of string theory may be provided by embedding the theory in the matrix of primordial spin structures given in Table 1 . This matrix constitutes a special application of the hypernumber idea, one that delivers a highly specific reckoning of primordial spin action. The topodimensional array of four fundamental spinors (shown on the principal diagonal of the matrix) can be directly associated with the four types of gauge bosons found in nature. The gauge-boson correlates of Table 1 are displayed in Table 5. What is the basis of these correlations?

\begin{tabular}{|c|c|c|c|}
\hline $\mathrm{G}$ & $\mathrm{G} / \mathrm{g}$ & $\mathrm{G} /(\mathrm{W}, \mathrm{Z})$ & $\mathrm{G} / \gamma$ \\
\hline $\mathrm{g} / \mathrm{G}$ & $\mathrm{g}$ & $\mathrm{g} /(\mathrm{W}, \mathrm{Z})$ & $\mathrm{g} / \gamma$ \\
\hline$(\mathrm{W}, \mathrm{Z}) / \mathrm{G}$ & $(\mathrm{W}, \mathrm{Z}) / \mathrm{g}$ & $\mathrm{W}, \mathrm{Z}$ & $(\mathrm{W}, \mathrm{Z}) / \gamma$ \\
\hline$\gamma / \mathrm{G}$ & $\gamma / \mathrm{g}$ & $\gamma /(\mathrm{W}, \mathrm{Z})$ & $\gamma$ \\
\hline
\end{tabular}

Table 5. Spin matrix of gauge bosons. $\mathrm{G}$ is the graviton; $\mathrm{g}$ is the strong gauge boson; $\mathrm{W}, \mathrm{Z}$ is the weak gauge boson particle pair; and $\gamma$ is the photon

We know that Table 1 signifies a process of generation in which higher topological dimensions evolve from lower ones. The facts of particle evolution lend themselves to straightforward, one-to-one correlation with this process. The first force particle to "freeze out" of the Big Bang's hot primordial soup is the hypothesized graviton, G. The graviton of Table 5 is associated with $\varepsilon_{\mathrm{D} 0}$, the zerodimensional sub-lemniscatory action of Table 1 , which can be written $\varepsilon_{\mathrm{D} 0}(\hbar / 2)$ to give expression to subatomic particle spin; thus, $G \equiv \varepsilon_{\mathrm{D} 0}(\hbar / 2)$. Next to separate itself from the primordial chaos is the strong gauge boson, $\mathrm{g}$, and we relate it to $\varepsilon_{\mathrm{D} 1}$ lemniscatory action, writing $\mathrm{g} \equiv \varepsilon_{\mathrm{D} 1}(\hbar / 2)$. Then the weak force emerges, given by the boson pair $\mathrm{W}$ and $\mathrm{Z}$, which we identify with $\varepsilon_{\mathrm{D} 2}(\hbar / 2)$. When the three orders of lower-dimensional gauge bosons have "frozen out," what remains is $\gamma$, the photon, topodimensionally expressed as $\varepsilon_{\mathrm{D} 3}(\hbar / 2)$.

If the principal terms or "fundamental tones" of the Table-5 matrix give the four gauge bosons, what is the significance of the "overtone-undertone" couplings appearing off the principal diagonal? In Table 1, these are the topodimensional enantiomorphs whose synsymmetric fusions drive the process of dimensional generation. The overtone-undertone couplings appear in Table 5 as enantiomorphically related boson ratios. It is from their interactions that the primary gauge bosons emerge. In phenomenological string theory, boson-ratio interaction not only accounts for the generation of the four kinds of gauge bosons, but also for the production of the 12 fermions of the standard model. The six pairs of ratios involved in distilling the bosons likewise interact to yield the six pairs of fermions (three lepton pairs and three quark pairs). Geometrically speaking, the fermions function as "dimensional bounding elements," local features of global 
bosonic dimensionality, with local and global aspects intimately interwoven. Needless to say, fermion generation requires clarification, but I will not elaborate further on it here (see Cosmos). What I will elaborate on is the specific course of boson generation that traces the evolution of nature's fundamental forces.

While Table 5 can be read as a process of dimensional generation, what we acknowledged earlier for Table 1 also applies to it: if Table 5 is taken by itself without reading change into it, the Table provides a merely static picture of particle relationships, a snapshot that misses the kinetic facts of cosmogony. Therefore, to see more clearly the developmental basis of the correlations suggested in Table 5, we must fill in the concrete details of how the four gauge bosons evolve in relation to each other. This is done in Table 6. 
Primordial Gravitation G

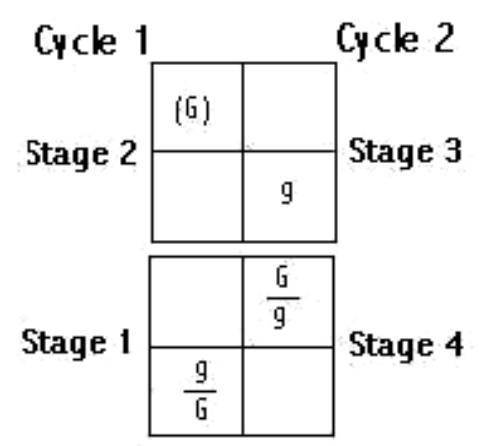

Evolution of Strong Force

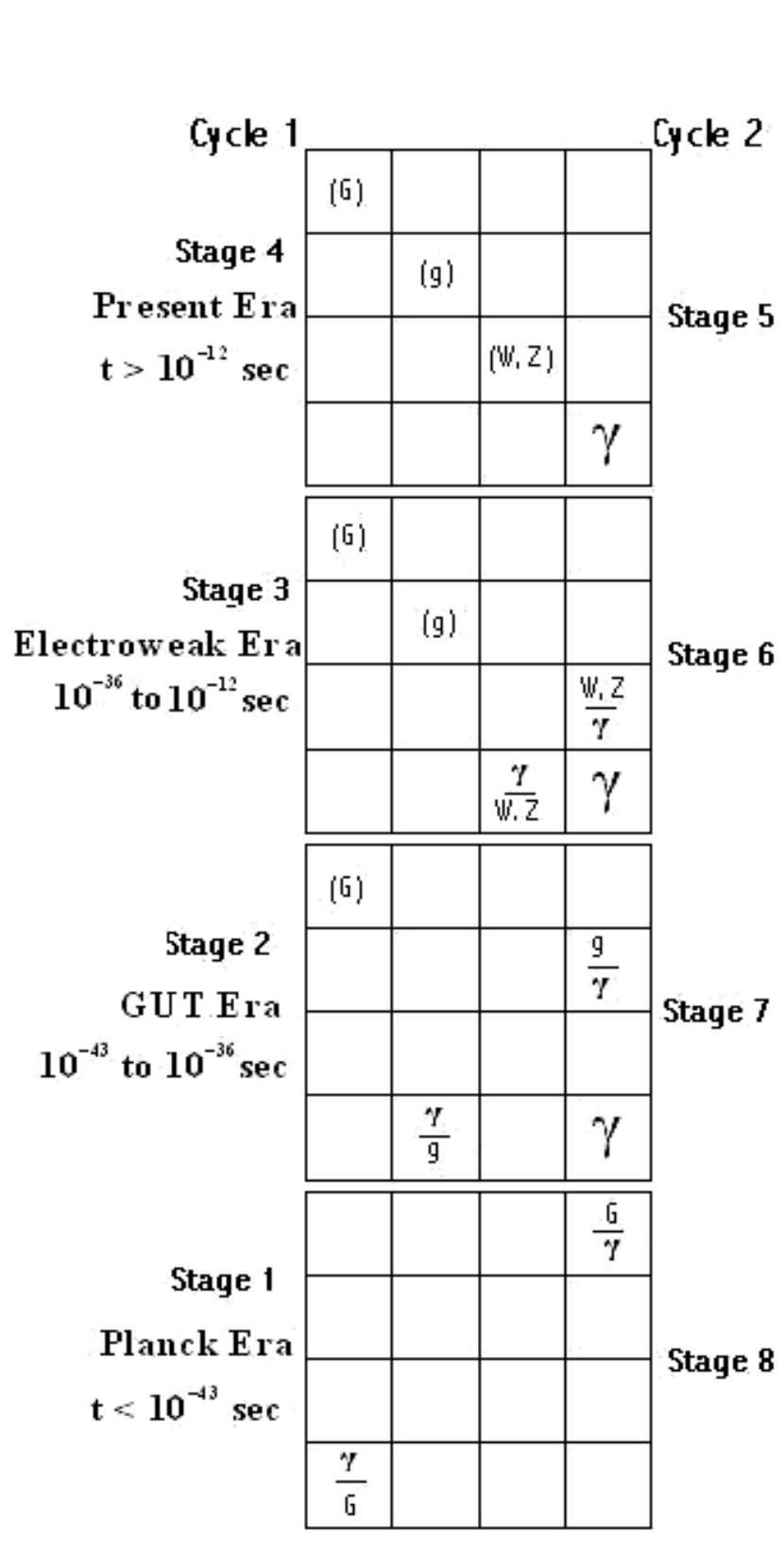

Evolution of Electromagn etism

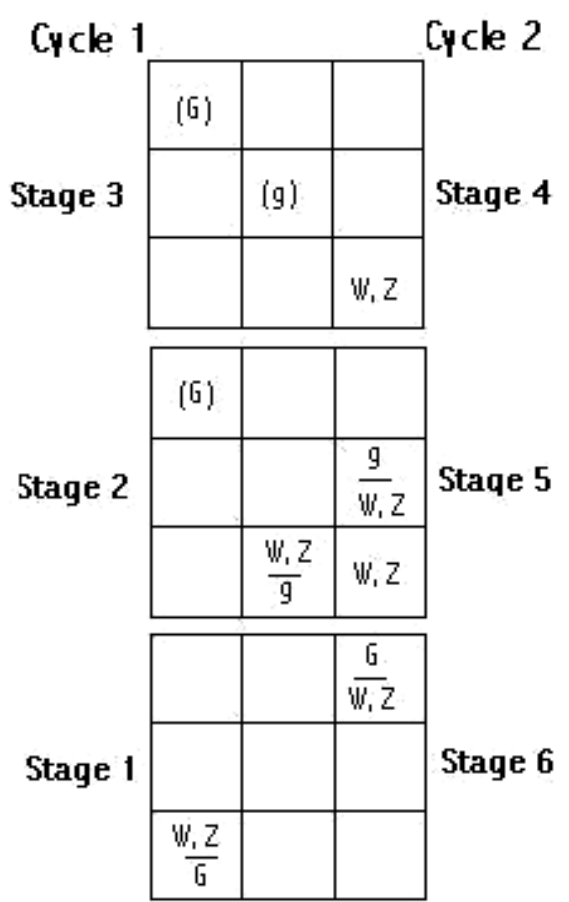

\section{Evolution of Weak Force}

Table 6. Spiral of particle evolution 
Table 6 presents the particulate counterparts of the cosmogonic events studied in Tables 3 and 4. The particles are not to be regarded as mere physical objects appearing in space. Each spinning particle (or, by the principle of complementarity, each wave) is in fact to be understood as a dynamic dimension unto itself, an archetypal action that combines matter and psyche in an intimate way. Phenomenologically, we can describe the particles as constituting lifeworlds, but it may be even better to view them as "braneworlds." For we are employing a certain kind of string theory, and, in the M-theoretic elaboration of string theory, "branes" enter the picture as higher-dimensional versions of strings. Therefore, if strings are taken as one-dimensional vibrations, branes (as in "membranes") can be vibrations of higher dimension. According to Greene, our whole universe might be conceived as a "braneworld" $(2004,386)$. What we are dealing with presently are four such universes.

With Table 6, we have a novel iteration of the cosmogonic spiral. Here the four braneworlds are represented in the four windings of the spiral. In section 4, we saw the consequence of considering cosmogony as a process of symmetry breaking: starting from symmetry, an effective dimensional rendering of nature's evolving forces cannot be realized. In the rendition signified by Table 6, the issue is resolved by regarding the dimensional generation of particles (waves, braneworlds) as resulting from the dialectical interplay of symmetry and asymmetry that we have called synsymmetry: the enantiomorphic fusion that creates braneworld symmetry in one winding carries with it the implication of an enantiomorphic diffusion giving rise to new asymmetry for the creation to takes place in the next winding.

We may compare the overall spiral pattern of cosmogony brought out in Tables 3, 4 and 6 to mainstream cosmological depictions. While most cosmologists now appear to agree that the three-dimensional universe was characterized early on by phases of expansion, there is less agreement on the fate of the universe. Will it continue to expand, or will it reverse itself and begin to contract? Cosmologists Paul Steinhardt and Neil Turok (2002) have addressed this question by offering a new version of the oscillating universe idea, one that has attracted much attention among astrophysicists. Taking their cue from string/M-theory, Steinhardt and Turok propose a cyclic model in which the universe undergoes countless rounds of expansion and contraction.

Our topo-phenomenological version of string theory suggests an evolving psychophysical cosmos that is not merely cyclical but spiralic. In agreement with Steinhardt and Turok, the notion of synsymmetry requires that the cosmic expansion we are currently experiencing in this three-dimensional Kleinian braneworld will be followed by a contraction, after which another period of expansion shall ensue. But the subsequent expansion will not just repeat the previous one. It will involve the opening up of a whole new dimension, including new forms of matter and a new force of nature beyond what appears in Table 6. I shall have more to say about this prospect before I conclude. What I presently want to emphasize is that the general picture of cosmic development I am proposing is neither of a simply open universe whose given dimensions expand indefinitely, nor of a closed universe featuring endless cycles of expansion and contraction. We must 
imagine instead an evolving cosmos whose contractions are the "labor pains" that accompany the birthing of new dimensional organisms. Each cosmic organism expands in its turn, only to experience cosmic contractions that pave the way for the next round of creative cosmic growth.

The windings of Table 6 unfold in close parallel with those of Tables $3 \mathrm{~b}$ and 4 , allowing us to see the connections among topodimensional, Taoist, and particulate descriptions of cosmogony. In the first winding, we have the graviton or gravitational wave, G. Given its equivalence to $\varepsilon_{\mathrm{D} 0}(\hbar / 2)$, we may take it to comprise the sub-lemniscatory zero-dimensional braneworld or vibrating universe. Rather than undergoing its own evolution, G functions purely as a carrier wave for the generation of higher-dimensional waves that initially reside as seeds within its zerodimensional field.

The second winding of the cosmogonic spiral concerns the generation of the strong nuclear force: $g \equiv \varepsilon_{\mathrm{D} 1}(\hbar / 2)$. The strong force corresponds to the lemniscatory braneworld consisting of one dimension. In stage 1 of this winding, we find a primordial matrix wherein $\mathrm{g}$ is manifested in nascent form, an embryonic particlewave supported by the overtone and undertone enantiomorphs of the $G$ carrier wave: $g / G$ and $G / g$, respectively. The projection of the one-dimensional braneworld has not yet occurred.

In advancing to the second stage of this projective cycle, $\mathrm{G}$ enantiomorphs fuse to bring symmetry to g. Thus gaining maturity, the strong-force universe expands, and, in the process, $\mathrm{G}$ is absorbed into the emergent structure of $\mathrm{g}$. This "annihilation" of G at the same time "freezes it out." We can see how this works from our analysis in sections 7 and 8.

Coincident with the projective potentiation of $g$ as the one-dimensional braneworld prevailing in stage $2, \mathrm{G}$ is subjected to a depotentiating projection. With its original vitality presently masked, it is manifested as an objectified dimension embedded within the larger, ostensibly objective universe of $\mathrm{g}$. It is in this form that $\mathrm{G}$ is "frozen out" in stage 2 (indicated in Table 6 by its enclosure within parentheses). So the primordial gravitational field that had served as carrier wave for the inchoate strong force in stage 1 is now in eclipse, with an attenuated remnant of it operating within the field of the mature strong force. There will be further occlusions of $\mathrm{G}$ in subsequent windings and this will eventuate in the attenuated form of gravitation known to us today-the form that conventional analysis cannot effectively reconcile with the other three forces of nature to yield a consistent theory of quantum gravity.

The events attending the transition to cycle 2 come as we might expect at this point in our investigation. In stage 3 , the gears shift from forward to backward, expansion to contraction, and the stage- 2 projection of a presumably objective strong-force universe is withdrawn via retrograde recognition of its sub-objective source. Then, in stage 4, the retrojection goes further and G emerges from eclipse, converging harmonically with $g$, thus sowing the seeds for the next winding.

With the opening of the third cosmogonic epoch, we have the generation of the weak boson pair: $\mathrm{W}$ and $\mathrm{Z} \equiv \varepsilon_{\mathrm{D} 2}(\hbar / 2)$. Topodimensionally, this entails the evolution of the two-dimensional Moebial braneworld. In the first stage of this 
winding, we find ourselves back in the primordial matrix of the gravitational force, with the embryonic weak force carried by the new enantiomorphs of G: (W, Z)/G and $\mathrm{G} /(\mathrm{W}, \mathrm{Z})$. Then, in stage 2 , these enantiomorphs fuse and symmetry is enhanced in a first projective expansion of the weak-force universe, coupled with a depotentiating projection of the gravitational force that freezes it out. At the same time, the potential for further weak-force development is carried by the enantiomorphs of the strong force, $(\mathrm{W}, \mathrm{Z}) / \mathrm{g}$ and $\mathrm{g} /(\mathrm{W}, \mathrm{Z})$. The final stage of cycle 1 completes the maturation of the weak force. Here strong-force enantiomorphs fuse, the strong force freezes out, and the weak force gains full symmetry with the projection of an ostensibly objective two-dimensional braneworld. Needless to say, all this is counteracted in shifting to the retrograde contractions of cycle 2: The projections of the weak force are withdrawn, the repressions of the strong and gravitational forces are lifted, and the three forces enter into harmony, sowing the seeds for the cosmogonic epoch to come.

The third round of particle generation is given in the Kleinian epoch that brings in the electromagnetic force, $\gamma \equiv \varepsilon_{\mathrm{D} 3}(\hbar / 2)$. By now the reader is well equipped to track the stages of projection shown for this winding. In Table 6 we see the development of $\gamma$ from its embryonic beginnings in the archaic gravitational matrix through three stages of expansion facilitated by enantiomorphic fusions of the lower-dimensional force particles. The process culminates in stage 4 with the projection of our visible three-dimensional universe-visible by means of the particle permitting us to view it, the photon.

Before considering the second cycle of the electromagnetic winding, I would like to discuss a feature of Table 6 that bears interesting implications for cosmogony. Only the electromagnetic winding is indexed chronologically by cosmogonic eras: the Planck era $\left(\mathrm{t}<10^{-43} \mathrm{sec}\right)$, the GUT era $\left(10^{-43}\right.$ to $\left.10^{-36} \mathrm{sec}\right)$, the Electroweak era $\left(10^{-36}\right.$ to $\left.10^{-12} \mathrm{sec}\right)$, and our present era $\left(t>10^{-12} \mathrm{sec}\right)$. Why are there no chronologically dated eras given for the lower-dimensional windings of the spiral? It is because the forms of time associated with them are not chronological. In section 4, I indicated that each topodimensional spinor is related to a different order not only of space but of time as well, though I did not elaborate on these differences. It does seem reasonable to hypothesize that different lifeworlds entail different orders of time, and that worlds independent of our three-dimensional universe are governed by forms of time qualitatively distinct from the linear time so familiar to us. While I have explored nonlinear temporality elsewhere (Rosen 2006), here I will only observe that our first cosmogonic winding surely defies chronological dating since it is utterly without time, expressing as it does the timelessness of the Tao (on the timeless aspect of the Tao, see Fagg 1985 and Butler-Bowdon 2012).

Now, the Kleinian winding of the cosmogonic spiral has special significance for us. It is our own winding. The dimensional context developed in it is the threedimensional world that frames our immediate perceptions and serves as the concrete ground of the very analysis carried out in this paper. The photon generated in the Kleinian epoch is indeed the primary means by which we make observations, conduct experiments, perform measurements, and generally interact with our 
environment. So, on the cosmogonic map, the words "you are here" might well have been marked for this epoch.

From our three-dimensional vantage point, we can describe the retrograde stages of the lower dimensions in a detached fashion, but we do not have that luxury when it comes to describing the second cycle of our own dimension. To unify the quantum gravitational field, the frozen-out, depotentiated braneworlds must be repotentiated in conjunction with the electromagnetic braneworld. Since the electromagnetic world is the analyst's own world, and since this is a psychophysical world rather than merely being physically "out there," the analyst cannot stand aloof from the process, treating it as something unfolding objectively before her. The analyst is here on the cosmogonic map, and he must participate accordingly.

To be sure, in entering cycle 2 , we have already taken the first step in this direction with our retrojective reflections on the psychophysical nature of our universe. Yet, in making the transition to stage 5 , our perspective remains strictly three-dimensional, as it was in stage 4 . What we need to do in the subsequent stages of this cycle is tangibly engage with the lower dimensional sub-objectivities as the repressions of them are lifted and their original potencies are restored. But just what would it mean for the scientist to forego his currently objectifying posture in favor of one that is deeply participatory?

\section{TOWARD A PARTICIPATORY COSMOGONY}

The philosopher of science Evelyn Fox-Keller calls for a new form of perception in scientific inquiry that she names "dynamic objectivity" $(1985,115)$. The old approach, she says, involves a "static objectivity" in which "the pursuit of knowledge...begins with the severance of subject from object" (117). In contrast,

dynamic objectivity aims at a form of knowledge that grants to the world around us its independent integrity but does so in a way that remains cognizant of, indeed relies on, our connectivity with that world. In this, dynamic objectivity is not unlike empathy, a form of knowledge of other persons that draws explicitly on the commonality of feelings and experience in order to enrich one's understanding of another in his or her own right. $(1985,117)$

Dynamic objectivity employs a type of awareness akin to the retrograde act of withdrawing projections. This is evidenced in Fox-Keller's citation of Piaget: "'Objectivity consists in...fully realizing the countless intrusions of the self in everyday thought and the countless illusions which result....So long as thought has not become conscious of self, it is prey to perpetual confusions between objective and subjective'" (117). According to Fox-Keller:

Dynamic objectivity is thus a pursuit of knowledge that makes use of subjective experience (Piaget calls it consciousness of self) in the interests of a more effective objectivity. Premised on continuity [of self and other], it recognizes difference between self and other as an opportunity for a deeper 
and more articulated kinship. The struggle to disentangle self from other is itself a source of insight-potentially into the nature of both self and other....To this end, the scientist employs a form of attention to the natural world that is like one's ideal attention to the human world: it is a form of love. The capacity for such attention, like the capacity for love and empathy, requires a sense of self secure enough to tolerate both difference and continuity. $(1985,117-18)$

Writing in the same vein, Fox-Keller adduces Ernest Schachtel's distinction between "autocentric" and "allocentric" perception. Whereas the former is "dominated by need or self-interest," the latter "is perception in the service of a love 'which wants to affirm others in their total and unique being.' It is an affirmation of objects as 'part of the same world of which man is a part,"' one which "permits a fuller, more 'global' understanding of the object in its own right" (119). Although Fox-Keller pays scant attention to phenomenological philosophy as such, the main thrust of her presentation is much in keeping with phenomenology's central aim, as expressed in its well-known slogan: "To the things themselves!" And it seems clear that the world shared by the "allocentric" observer and the objects that s/he observes is the lifeworld of phenomenology.

Fox-Keller helps us gain a better grasp of the new mode of scientific inquiry by offering a specific example of one of its premier practitioners: the Nobel prizewinning biologist, Barbara McClintock. In stark contrast to the detached, dispassionate attitude of the Cartesian scientist, McClintock speaks of obtaining an intimate feeling for the plants she works with: "I don't feel I really know the story if I don't watch the plant all the way along. So I know every plant in the field. I know them intimately, and I find it a great pleasure to know them"' (Fox-Keller 1985, 164). In another place, McClintock:

describes the state of mind accompanying the crucial shift in orientation that enabled her to identify chromosomes she had earlier not been able to distinguish: "I found that the more I worked with them, the bigger and bigger [the chromosomes] got, and when I was really working with them I wasn't outside, I was down there. I was part of the system....It surprised me because I actually felt as if I was right down there and these were my friends....As you look at these things, they become part of you. And you forget yourself." (McClintock quoted in Fox-Keller 1985, 165)

Fox-Keller observes that McClintock's vocabulary "is consistently a vocabulary of affection, of kinship, of empathy," an empathy that constitutes "the highest form of love: love that allows for intimacy without the annihilation of difference" (164). Here the word "love" is used "neither loosely nor sentimentally, but out of fidelity to the language McClintock herself uses to describe a form of attention, indeed a form of thought" (164).

Fox-Keller arrives at these conclusions: 
The crucial point for us is that McClintock can risk the suspension of boundaries between subject and object without jeopardy to science precisely because, to her, science is not premised on that division. Indeed, the intimacy she experiences with the objects she studies...is a wellspring of her powers as a scientist....In this world of difference, division is relinquished without generating chaos. Self and other, mind and nature survive not in mutual alienation, or in symbiotic fusion, but in structural integrity. $(1985,164-165)$

Finally, after recounting the goal of conventional science, Fox-Keller observes that, "To McClintock, science has a different goal: not prediction per se, but understanding; not the power to manipulate, but empowerment-the kind of power that results from an understanding of the world around us, that simultaneously reflects and affirms our connection to that world" (166).

In phenomenological terms, the world to which McClintock is connected in feeling and embodied empathy is the lifeworld. It is a world in which the dialectic of difference and identity is enacted through an intimate knowledge of other that requires and is inseparable from the knowledge of self (a "consciousness of self"). McClintock's "revolution that 'will reorganize...the way we do [scientific] research"' (Fox-Keller 1985, 172) depends upon descending from the Cartesian stratosphere and immersing ourselves in the psychophysical dimension wherein object and subject, symmetry and asymmetry, continuity and discontinuity mediate one another internally in an encompassing circular flow. We have not forgotten that the source of this phenomenological circulation lies in the ancient eddies of yin and yang.

Fox-Keller's "dynamic objectivity" as exemplified by McClintock is hardly the only instance of the burgeoning of a new dialectical science. The phenomenological initiative, begun early in the twentieth century, has been advanced by thinkers like Heelan (1983) and Gendlin (1991), who have proposed that the work of science not proceed from "stratospheric" perception, but from the intricacies of the lifeworld or lived body. A dialectical approach to science also is advocated by biophysicist Koichiro Matsuno (1995). Matsuno has called for a "dialogical" science that would supersede the old "monologue" carried on by the solitary Cartesian subject looking down upon the world from above. In Matsuno's vision, scientific activity would involve a community of subjects concretely engaged with each other in dynamic and generative negotiations. Whereas the Cartesian subject is anonymous, absent from the events that transpire, the participants in the dialectical community would function self-referentially to include themselves in the process (Matsuno exemplifies this by explicitly including himself as author in what he writes; 1995, 1998). Other important contributions come from Plamen Simeonov (2012), who has emphasized the need to devise first-person methodologies for the natural sciences; from Arran Gare (2013), with his insistence that science be grounded in a way that includes lived subjectivity; and from Louis Kauffman's (2015) reflections on how mathematical self-reference is related to topology and phenomenological philosophy. Still another contribution to emergent dialectical science is offered by the Jungian psychologist Nathan Schwartz-Salant (2007). Operating selfreferentially, Schwartz-Salant employs Merleau-Ponty and the Klein bottle in 
characterizing the deep psychodynamics of human relationships, and he likens the fields operative in these paradoxical interactions to field processes in fundamental physics (see also Schwartz-Salant 2017).

What we require in the present context is a dialectical cosmogony. In this approach, the analysis of cosmogony is situated within cosmogony itself ("we are part of the world we are trying to know," says Gare; 2013, 25). The unquestioned objective stance analysts have tended to take toward cosmic evolution is in fact characteristic of the fourth and final stage of projection in the Kleinian epoch of dimensional generation. This is the stage of development in which we assume that cosmogonic events are "objectively out there," and that we analysts are detached from them, with our lived subjectivity playing no role in what we see. In this stage, the common sense notion of an external universe developing on its own is so compelling that it seems absurd for us to think otherwise. But, in advancing to the stages of cycle 2 , the point comes home to us that we are indeed intimate participants in the story of cosmic creation. Thus entering into cosmogonic process, the classical posture of analysis gives way to a phenomenological one in which our own process of development plays an integral role. In the act of inwardly grasping the transformation of the cosmos, the analyst surpasses the projective construction of herself as an isolated onlooker and takes part in the drama of creating a world. So, if the cosmos is self-evolving, the self of the analyst figures essentially in the reflexive enactment of this process.

But let me try to be clearer about what the involvement of the analyst specifically entails. The proposition I venture to suggest is that a fully reflexive analysis of cosmogony requires that, in investigating the stages of cosmogonic retrojection, the analyst must gain palpable awareness of his or her own stages of development. Only then can the link to cosmic development be realized in its existential immediacy, since, only then would the analyst realize cosmic transformation as a self-transformation, not just a transformation of what is other. Of course, the analytical self in question cannot merely be that of a particular individual. The self that participates in the archetypal processes of cosmic creation must function archetypally. Yet it seems we need to begin with the particular person if the process is to be grounded in existential reality. Presumably, in the course of deeply exploring his or her own past, the analyst would cross a threshold and her personal being would shade into the transpersonal. The transpersonal psychiatrist Stanislav Grof expressed a similar idea in describing the transformation of awareness that can occur in the act of re-experiencing the "perinatal" stages of development, those occurring around the time of birth: "All we can say is that somewhere in the process of confrontation with the perinatal level of the psyche, a strange qualitative Moebius-like [!] shift seems to occur in which deep selfexploration of the individual unconscious turns into a process of experiential adventures in the universe-at-large" $(1985,36)$.

In the projective moment of cosmogony, it may well seem a flight of fancy to link the stages of human development to those of the cosmos as a whole. The phenomenological response to this incredulity extends the biological dictum that "ontogeny recapitulates phylogeny" to the field of physics and says, ontogeny recapitulates cosmogony. For, if it is true that we participate in the story of creation 
in a full-fledged way, it would seem that our own history would be inseparable not only from that of the broader biological world but from nature at large. Evidently then, when we move backward through the stages of cycle 2 to gain "allocentric" awareness of the braneworlds belonging to nature's archaic past, it seems we must work through our own archaic past if we are to apprehend those worlds in the most concrete, immediate, and deeply reflexive way.

Embryological research certainly appears to support the idea that the early development of the human individual mirrors the development of the species as a whole. In fact, my earlier work links ontogeny and phylogeny explicitly, and in a detailed way (Rosen 2006). Presently, it is ontogeny and cosmogony that must be linked. Some theorists have broadly speculated that the universe functions as a giant hologram (Bohm 1980, 189). Such a cosmos should possess a fractal pattern of selfsimilarity, with the structure and development of the whole being mirrored recursively on every scale of magnitude down to the smallest part. Then-if probing the early history of an individual member of the phylogenetic order opens out into phylogeny as a whole-it is perhaps not unreasonable to hypothesize a deeper stratum of self-similarity involving the history of the cosmos to which we belong. Relevant in this regard is the vision of physicist Lee Smolin:

Living things share in some ways, and extend in other ways, the basic properties of non-equilibrium self-organized systems that seem to characterize the universe on every scale, from the cosmos as a whole to the surface of planets....If life, order and structure are the natural state of the cosmos itself, then our existence, indeed our spirit, might finally be comprehended as created naturally, by the world, rather than unnaturally and in opposition to it. $(1997,160)$

In a similar vein, biophysical theorist Hector Sabelli asserts that "the continuity of evolution requires that the same fundamental forms must be expressed at the physical, biological, and psychological levels of organization" $(2005,431)$. This is consistent, of course, with the psychophysical nature of cosmogonic process.

In The Self-Evolving Cosmos, I attempted to spell out more specifically the manner in which the analyst's own development can be linked to that of the cosmos at large. But I can go no further here if this already lengthy paper is to stop short of becoming a book!

Let me conclude by returning to the issue raised near the end of section 7 . There I noted that the guiding principle of synsymmetry implies the birth of a higher-dimensional lifeworld surpassing the Kleinian. The question I posed was how we can come to know this four-dimensional reality in a tangible way. What I indicated is that going beyond an abstract three-dimensional analysis of the fourth dimension necessitates the dimensional evolution of our analytic framework itself. At that point, it was already implicit that the backward movement through the second Kleinian cycle brings this evolution about. We knew that, in the course of cycle 2, lower-dimensional organisms rise from obscurity, and-operating in synchrony with the Kleinian organism - sow the seeds for the meta-Kleinian, fourdimensional framework. But we knew this only abstractly, since our way of knowing 
was itself restricted to the three-dimensional context. So the obstacle to tangibly apprehending the fourth dimension is our inability to grasp it in a manner transcending the limits of our three-dimensional frame of analysis. Clearly the theoretical anticipation of completing the second Kleinian cycle is no substitute for completing it in actuality. How can the latter be achieved? I suggest it can happen by adopting the reflexive phenomenological posture intimated above. Here we participate allocentrically with the lower-dimensional organisms of cycle 2, investigating the stages of development as stages in our own development, experiencing the transformations occurring as self-transformations. It is when this process is brought to fruition that a new winding of the cosmogonic spiral opens up and a whole new world is introduced-a world we will have come to know substantively by the dimensional expansion of our capacity for knowing.

While my own epistemic capacity remains distinctly three-dimensional, let me offer for what it is worth a provisional, still quite abstract impression of what may be in store. In the wider turning of the spiral, a new and more dialectically intricate, four-dimensional braneworld would come into play beyond the Kleinian world, a topological action pattern laid out in $5 \times 5$ matrices. In this novel world, a new force of nature presumably will emerge, along with new forms of matter. A fresh configuration of yin and yang should express this transformation, symbolized by four lines instead of three (the "quadrigram" would surpass the trigram). In the language of the I Ching, the "forces of the coming year" would thus "unroll." And as cosmogony would advance once more in the epoch to come, the music of the dimensional spheres should resound in a previously unheard register, audible only to those with the capacity to hear it.

\section{REFERENCES}

Angeles, Peter A. 1981. Dictionary of Philosophy. New York: Barnes and Noble. Barr, Stephen. 1964. Experiments in Topology. New York: Dover.

Bohm, David. 1980. Wholeness and the Implicate Order. London: Routledge and Kegan Paul.

Butler-Bowdon, Tom. 2012. "Introduction." In Lao Tzu's Tao Te Ching, vii-xxiv. West Sussex, UK: Capstone Publishing.

Chia, Mantak and William U. Wei. 2009. Living in the Tao. Rochester, VT: Destiny Books.

Fagg, Lawrence W. 1985. Two Faces of Time. Wheaton, IL: The Theosophical Publishing House.

Fowler, Jeaneane. 2005. An Introduction to the Philosophy and Religion of Taoism. East Sussex, UK: Sussex Academic Press.

Fox-Keller, Evelyn. 1985. Reflections on Gender and Science. New Haven, CT: Yale University Press.

Gare, Arran. 2013. "Overcoming the Newtonian Paradigm: The Unfinished Project of Theoretical Biology from a Schellingian Perspective." Progress in Biophysics and Molecular Biology 113(1):5-24. 
Gendlin, Eugene T. 1991. “Thinking Beyond Patterns: Body, Language, and Situations." In The Presence of Feeling in Thought, edited by Bernard denOuden and Marcia Moen, 27-189. New York: Peter Lang.

Godwin, Joscelyn, Ed. 1989. Cosmic Music. Rochester, VT: Inner Traditions.

- - . 1992. Harmony of the Spheres. Rochester, VT: Inner Traditions.

Greene, Brian. 1999. The Elegant Universe. New York: W. W. Norton.

- - - 2004. The Fabric of the Cosmos. New York: Alfred A Knopf.

Grigg, Ray. 1994. The Tao of Zen. Rutland, VT: Tuttle Publishing.

Grof, Stanislav. 1985. "Modern Consciousness Research and Human Survival." ReVision 8:27-39.

Haase, Rudolf. 1989. "Harmonics and Sacred Tradition." In Cosmic Music, edited by Joscelyn Godwin, 91-110. Rochester, VT: Inner Traditions.

Heelan, Patrick A. 1983. Space-Perception and the Philosophy of Science. Berkeley, CA: University of California Press.

Hulse, David Allen. 2002. The Eastern Mysteries. St. Paul, MN: Llewellyn Publications.

Jung, Carl Gustav. 1970. Mysterium Coniunctionis. Vol. 14 of Collected Works, translated by R. F. C. Hull. Princeton, NJ: Princeton University Press.

Kauffman, Louis. 2015. "Self-Reference, Biologic and the Structure of Reproduction." Progress in Biophysics and Molecular Biology 119(3):382-409.

Lai, Whalen. 1980. "The I-Ching and the Formation of the Hua-Yen Philosophy." Journal of Chinese Philosophy 7:245-258.

Matsuno, Koichiro. 1995. "Use of Natural Languages in Modeling Evolutionary Processes." Proceedings of the $14^{\text {th }}$ International Congress of Cybernetics (International Association of Cybernetics, Namur, Belgium), 477-82.

- - . 1998. "Space-Time Framework of Internal Measurement." In Computing Anticipatory Systems, AIP Conference Proceedings 437, edited by D. M. Dubois, 101-15. Woodbury, NY: American Institute of Physics.

May, Reinhard. 1996. Heidegger's Hidden Sources. Abingdon, UK: Routledge.

Merleau-Ponty, Maurice. 1964. "Eye and Mind." In The Primacy of Perception, edited by James M. Edie, 159-90. Evanston, IL: Northwestern University Press.

-_- 1968. The Visible and the Invisible, translated by Alphonso Lingis. Evanston, IL: Northwestern University Press.

Musès, Charles. 1968. "Hypernumber and Metadimension Theory." Journal of Consciousness Studies 1:29-48.

———. 1976. "Applied Hypernumbers: Computational Concepts." Applied Mathematics and Computation 3:211-226.

Ozaki, Makoto. 2001. Individuum, Society, and Humankind. Leyden: Brill.

Pagels, Heinz R. 1985. Perfect Symmetry. New York: Bantam.

Priest, Stephen. 2003. Merleau-Ponty. London: Routledge.

Rosen, Steven M. 1975. "Synsymmetry." Scientia 110:539-549.

- - . 1994. Science, Paradox, and the Moebius Principle. Albany: State University of New York Press.

-_- 1995. "Pouring Old Wine into a New Bottle." In The Interactive Field in Analysis, edited by Murray Stein, 121-41. Wilmette, IL: Chiron.

- - 2004. Dimensions of Apeiron. Amsterdam: Editions Rodopi/Brill.

- - 2006. Topologies of the Flesh. Athens, OH: Ohio University Press. 
-——. 2008a. The Self-Evolving Cosmos. Hackensack, NJ: World Scientific.

—_- 2008b. "Quantum Gravity and Phenomenological Philosophy." Foundations of Physics 38:556-582.

-—_. 2014. Dreams, Death, Rebirth. Asheville, NC: Chiron.

—_- 2015. "Why Natural Science Needs Phenomenological Philosophy." Progress in Biophysics and Molecular Biology 119(3):257-69.

Rucker, Rudolph. 1977. Geometry, Relativity, and the Fourth Dimension. New York: Dover.

Ryan, Paul. 1993. Video Mind/Earth Mind: Art, Communications, and Ecology. New York: Peter Lang.

Sabelli, Hector. 2005. Bios: A Study of Creation. Hackensack, NJ: World Scientific. Schwartz-Salant, Nathan. 2007. The Black Nightgown. Wilmette, IL: Chiron.

_- - 2017. The Order-Disorder Paradox. Berkeley, CA: North Atlantic Books.

Simeonov, Plamen L. 2012. Integral Biomathics: Tracing the Road to Reality. Heidelberg: Springer.

Smith Jr., Kidder, Peter K. Bol, Joseph A. Adler, and Don J. Wyatt. 1990. Sung Dynasty Uses of the I Ching. Princeton, NJ: Princeton University Press.

Smolin, Lee. 1997. The Life of the Cosmos. Oxford: Oxford University Press.

-_- 2006. The Trouble With Physics. Boston: Houghton Mifflin.

Steinhardt, Paul J., and Neil Turok. 2002. "A Cyclic Model of the Universe." Science 296:1436-39.

von Franz, Marie-Louise. 1974. Number and Time. Evanston, IL: Northwestern University Press.

Woit, Peter. 2006. Not Even Wrong. New York: Basic Books. 